Review began 02/24/2022 Review ended 03/03/2022 Published 03/07/2022

๑) Copyright 2022

Tarrar et al. This is an open access article distributed under the terms of the Creative Commons Attribution License CC-BY 4.0., which permits unrestricted use, distribution, and reproduction in any medium, provided the original author and source are credited.

\section{Current Status of Monoclonal Antibodies-Based Therapies in Castration-Resistant Prostate Cancer: A Systematic Review and Meta-Analysis of Clinical Trials}

Talha Azam Tarrar ${ }^{1}$, Muhammad Yasir Anwar ${ }^{2}$, Muhammad Ashar Ali ${ }^{3}$, Memoona Saeed ${ }^{4}$, Sana Rehman ${ }^{5}$, Shammas F. Bajwa ${ }^{6}$, Tooba Ayub ${ }^{7}$, Haleema Javid ${ }^{8}$, Rimsha Ali ${ }^{9}$, Alaa Irshad ${ }^{10}$, Wajeeha Aiman ${ }^{3}$

1. Internal Medicine, Nottingham University Hospitals NHS Trust, Nottingham, GBR 2. Internal Medicine, BronxCare Health System, Bronx, USA 3. Internal Medicine, Beth Israel Deaconess Medical Center, Harvard Medical School, Boston, USA 4. Internal Medicine, Mayo Hospital, Lahore, PAK 5. Internal Medicine, Shaikh Khalifa Bin Zayed AlNahyan Medical and Dental College, Lahore, PAK 6. Internal Medicine, AdventHealth Orlando, Orlando, USA 7. Internal Medicine, MacNeal Hospital, Berwyn, USA 8. Internal Medicine, Rawalpindi Medical University and Allied Hospitals, Rawalpindi, PAK 9. Internal Medicine, Rawalpindi Medical University, Rawalpindi, PAK 10. Internal Medicine, Allama Iqbal Medical College, Lahore, PAK

Corresponding author: Muhammad Ashar Ali, asharalianwar94@gmail.com

\section{Abstract \\ Background}

Multiple patients with prostate cancer become resistant to castration therapies, which is termed castrationresistant prostate cancer (CRPC).

\section{Purpose}

The purpose of this review is to assess the status of efficacy ( $\geqslant 50 \%$ decline in prostate-specific antigen (PSA), progression-free survival (PFS), and overall survival (OS)) and safety (grade 3-4 adverse effects) of monoclonal antibodies in CRPC.

\section{Data source}

We searched databases including PubMed, Embase, Cochrane, Web of Science, and ClinicalTrials.gov.

\section{Results}

Hazard ratios of PFS and OS were $0.77\left(95 \% \mathrm{CI}=0.69-0.87, \mathrm{I}^{2}=53 \%\right)$ and $0.98\left(95 \% \mathrm{CI}=0.86-1.11, \mathrm{I}^{2}=40 \%\right)$, respectively, in the favor of monoclonal antibodies as compared to placebo. Risk ratio (RR) of $>50 \%$ decline in PSA was 1.99 (95\% CI $\left.=0.97-4.08, \mathrm{I}^{2}=53 \%\right)$ in favor of monoclonal antibodies. Pooled incidence of $>50 \%$ decline in PSA levels was $15 \%$ ( $\left.95 \% \mathrm{CI}=0.1-0.23, \mathrm{I}^{2}=83 \%\right), 29 \%$ (95\% CI $\left.=0.14-0.51, \mathrm{I}^{2}=93 \%\right), 63 \%$ (95\% CI $\left.=0.49-0.76, \mathrm{I}^{2}=77 \%\right)$, and $88 \%\left(95 \% \mathrm{CI}=0.81-0.93, \mathrm{I}^{2}=0 \%\right)$ in single, two, three, and four-drug regimens, respectively.

\section{Conclusion}

Monoclonal antibodies are well tolerated and showed better PFS as compared to placebo. However, OS was only improved with ipilimumab. Denosumab delayed skeletal-related adverse events as compared to zoledronic acid. More multicenter double-blind clinical trials may be needed to confirm these results.

Categories: Urology, Oncology, Therapeutics

Keywords: castration-resistant prostate cancer, meta-analysis, systematic review, checkpoint inhibitors, monoclonal antibodies, prostate cancer

\section{Introduction}

Prostate cancer is the second most common cause of cancer deaths in men after lung cancer in the United States with both aggressive and slow-growing types identified. More than $20 \%$ of the newly diagnosed cases of cancer are prostate cancer [1]. The new cases and estimated deaths for prostate cancer reported in the US in 2019 were 174,650 and 31,620, respectively, with an increase in the trend seen in 2020 with 191,930 new cases and 33,330 estimated deaths [1,2]. Globally, 1,276,106 new cases were estimated in 2018. Developed countries have higher incidence probably due to better use of diagnostic testing [3].

The various modalities that continue to be the mainstay of treatment for prostate cancer are surgical 
(prostatectomy), hormonal (gonadotropin-releasing hormone agonist or antagonist, androgen deprivation), and radiation (external beam radiotherapy, brachytherapy) [4-6]. However, surgical/chemical castration is required for most patients with metastatic disease. The progression of the carcinoma with or without metastasis despite castration therapy (androgen deprivation therapy) is termed as castrate-resistant or hormone-resistant cancer and is characterized by rising prostate-specific antigen (PSA) levels with castrate range of testosterone $(<50 \mathrm{ng} / \mathrm{dl}$ or $<1.7 \mathrm{nmol} / \mathrm{l})[6-9]$.

Chemotherapy agents including taxanes, bisphosphonates, immunotherapy agents, and poly (ADP-ribose) polymerase- 1 inhibitors have shown anti-tumor activity in patients with castration-resistant prostate cancer (CRPC). Taxane with prednisone is the most common treatment used for CRPC. Despite these treatment options, the prognosis and quality of life of these patients are very poor. There is still room for more combination therapies for the treatment of CRPC, especially for patients who do not tolerate and/or are refractory to first-line therapies [10-13].

In recent years, monoclonal antibodies have shown promising results in clinical trials. Monoclonal antibodies have been evaluated for their efficacy in CRPC due to their targeted action on various tumor factors that help control cancer progression [4]. The most common antibodies studied include bevacizumab (anti-vascular endothelial growth factor (VEGF)), which decreases angiogenesis and improves vessel penetration of cytotoxic agents like taxanes when used in combination [10,11]. Cixutumumab and ramucirumab act against insulin-like growth factor-1 receptor (IGF-1R)/vascular endothelial growth factor receptor (VEGFR) and can prevent tumor growth. Other monoclonal antibodies, including siltuximab, abituzumab, trastuzumab, and cetuximab, bind to interleukin-6, integrin alpha-V, human epidermal growth factor receptor 2 (HER2), and epidermal growth factor receptor (EGFR), respectively [12-15]. Checkpoint inhibitors including nivolumab (anti-programmed cell death protein 1 (PD-1)), pembrolizumab (anti-PD-1), and ipilimumab (anti-cytotoxic T-lymphocyte-associated antigen-4 (CTLA-4)) are also tested in clinical trials for anti-tumor activity against CRPC $[16,17]$. While several of these immunotherapies are under evaluation in clinical trials, denosumab is the major monoclonal antibody approved by the FDA for metastatic bone lesions in CRPC [18].

The aim of this systematic review and meta-analysis is to assess the efficacy and safety of monoclonal antibodies alone or in combination with chemotherapy drugs in CRPC.

\section{Materials And Methods}

In conducting this systematic review and meta-analysis, we followed a prespecified protocol registered on the International Prospective Register of Systematic Reviews (PROSPERO) (registration number: CRD42021230102). The protocol was made according to the guidelines established by Cochrane [19] and PRISMA-P (Preferred Reporting Items for Systematic Review and Meta-Analysis Protocols) [20].

\section{Search strategy}

A literature search was performed on PubMed, Embase, Web of Science, Cochrane Library, and ClinicalTrials.gov with Medical Subject Heading (MeSH) and Emtree terms “monoclonal antibodies" and "castration-resistant prostate cancer." The search was made from the inception of literature till March 20, 2021, by following the PICO framework (Appendix) [21].

\section{Inclusion and exclusion criteria}

We included all clinical trials that provided safety and efficacy data in clinical terms, i.e., objective response $(\mathrm{OR})$, complete response (CR), partial response (PR), $\geqslant 50 \%$ decline in PSA, progression-free survival (PFS), overall survival (OS), and grade 3-4 adverse effects. We excluded all preclinical studies, case reports, metaanalyses, review articles, observation studies, and clinical studies irrelevant to the study question.

\section{Study selection}

Two researchers (WA and TAT) independently reviewed the articles identified through initial search and screened them based on inclusion and exclusion criteria. The differences were addressed by a third researcher (MAA).

\section{Data extraction}

Data were extracted by two authors (MS and MYA). The data were extracted for the characteristics of the study, baseline characteristics of participants, treatment drugs, efficacy measures, and toxicity (grade $\geqslant 3$ adverse effects).

\section{Risk of bias assessment}

Two researchers (SR and SFB) assessed the risk of bias in randomized clinical trials (RCTs) selected for final inclusion by using the Risk of Bias 2 (RoB 2) tool for risk of bias assessment in RCTs [22]. The third researcher (MAA) addressed the differences. 


\section{Cureus}

\section{Statistical analysis}

The meta-analysis was performed using the "R" programming language. We used the "meta" package in $\mathrm{R}$ for our data analysis [23]. A random-effects model was used, irrespective of the heterogeneity, to keep our results consistent and applicable. All analyses used the DerSimonian-Laird estimator to calculate betweenstudy variance. The risk ratios were pooled using the Mantel-Haenszel method. For studies with zero events in any of the arms, a continuity correction of 0.5 was used. Standard errors and other calculations were done using a $95 \%$ confidence interval. For pooling of the results, all the studies were included even if they have zero events in both arms. To estimate the heterogeneity, $\mathrm{I}^{2}$ was used.

\section{Results}

A total of 3,069 articles were identified with 424 articles from PubMed, 2,427 articles from Embase, 49 articles from Web of Science, 60 articles from Cochrane, and 109 articles from ClinicalTrials.gov. These articles were analyzed by the researchers and 416 articles were removed as duplicates. A total of 2,221 articles were excluded in the first screening based on exclusion criteria. Full texts of 432 articles were reviewed. Eight RCTs ( $N=6,227)[13,24-30]$ and 18 non-randomized clinical trials (NRCTs, $N=920$ ) [10,15,31-41] were included based on prespecified inclusion criteria (Figure 1).
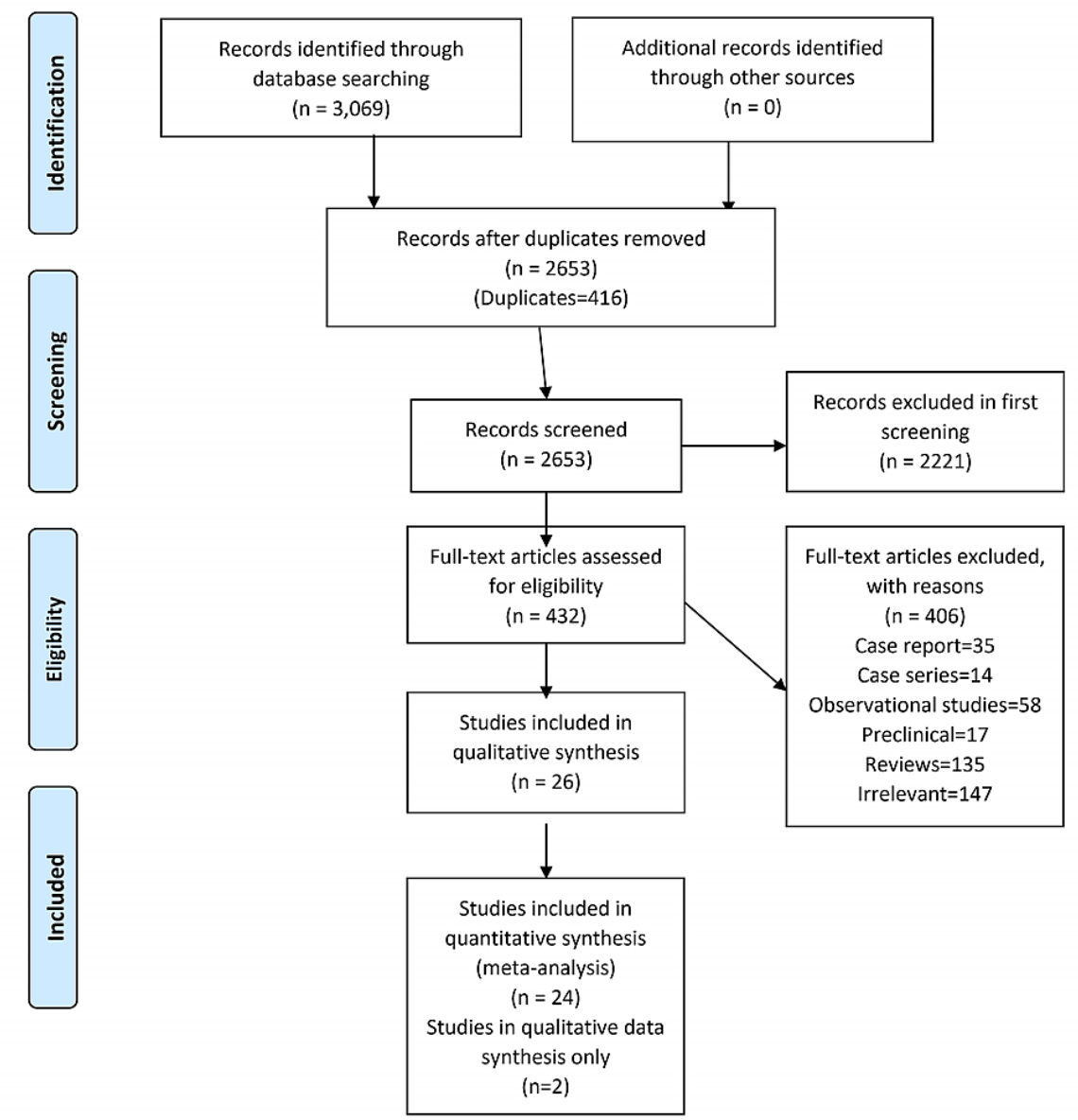

FIGURE 1: Flow chart of literature search.

\section{Risk of bias}

The risk of bias was low in double-blinded RCTs except for open-label RCT conducted by Hussain et al. (2015) [30] (Figure 2). 


\section{Cureus}

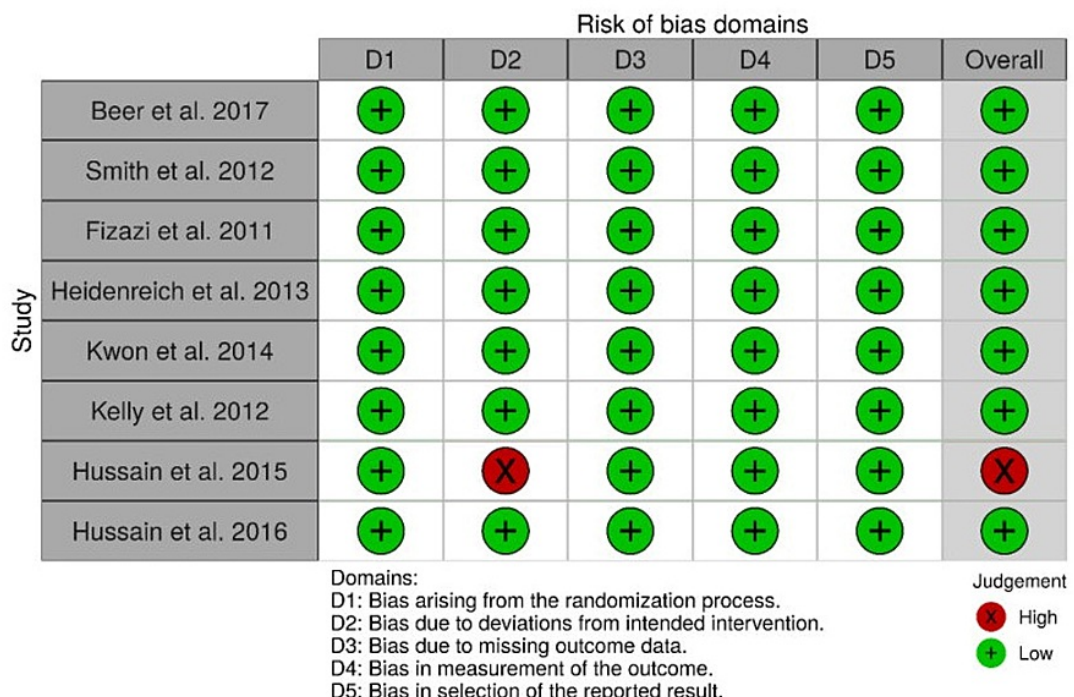

Bias arising from the randomization process Bias due to deviations from intended interventions

Bias due to missing outcome data

Bias in measurement of the outcome

Bias in selection of the reported result

Overall risk of bias

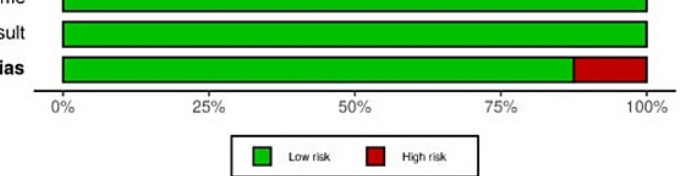

FIGURE 2: Risk of bias assessment with Risk of Bias 2 (RoB 2) tool.

Studies $[12,13,24-30]$

\section{Monoclonal antibodies vs. placebo}

In six clinical trials $(\mathrm{N}=4,194)$ [13,24-30], monoclonal antibodies were given to 2,225 participants while placebo was given to 1,969 participants. Standard of care (SOC) including luteinizing hormone-releasing hormone agonist/antagonist was given to 180 patients in the study by Hussain et al. [30]. The median ages of participants were $\geqslant 65$ years in RCTs. Baseline characteristics of participants are given in Table 1 .

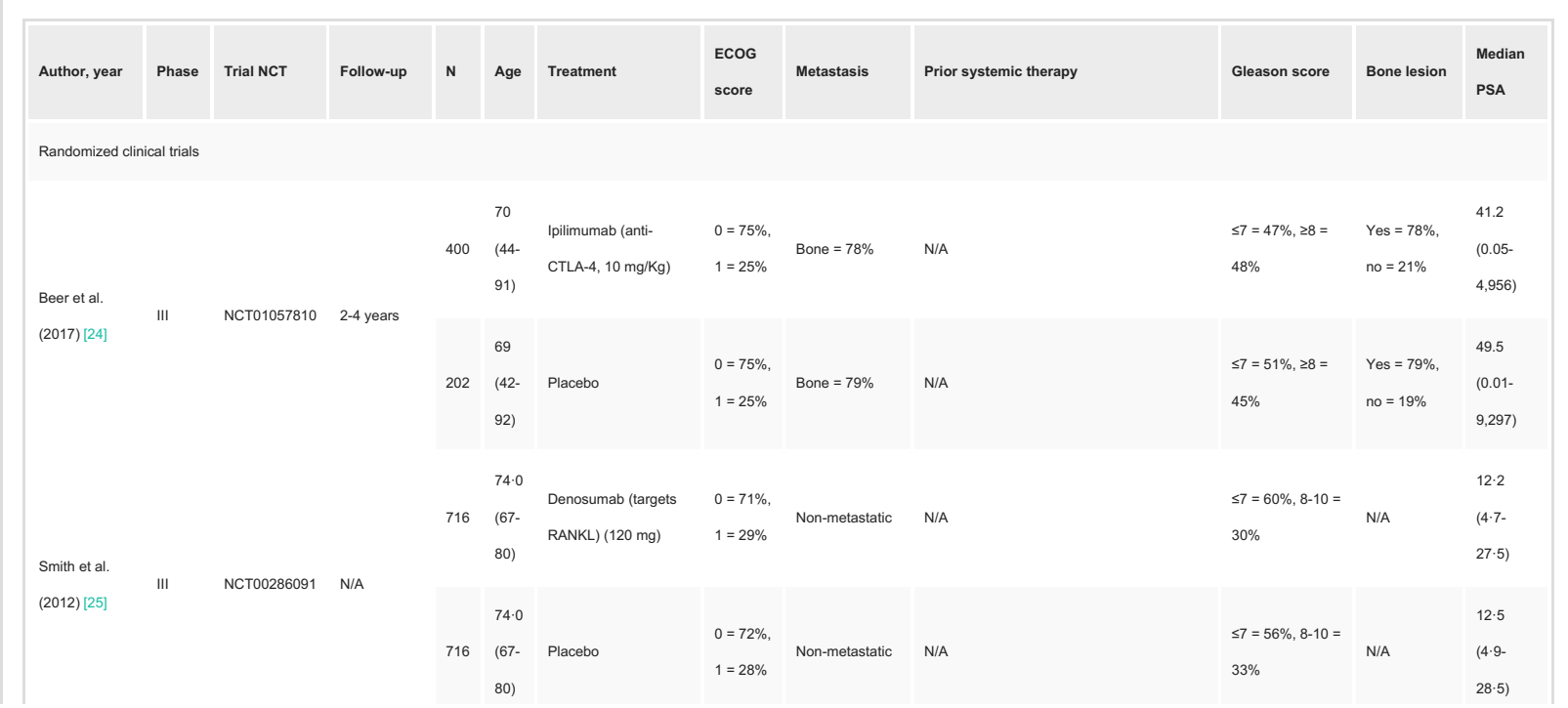




\section{Cureus}

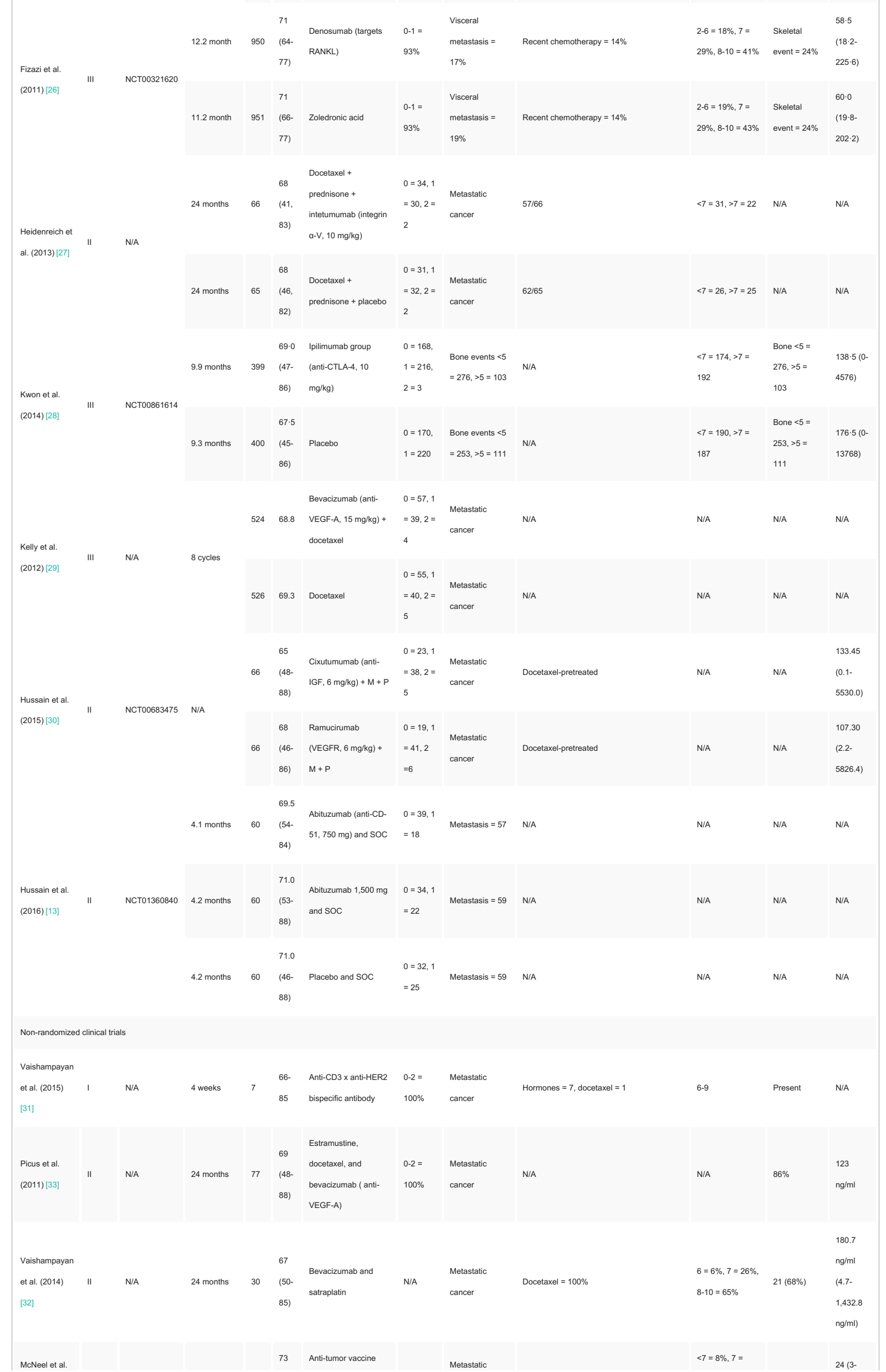




\section{Cureus}

\begin{tabular}{|c|c|c|c|c|c|c|c|c|c|c|c|c|}
\hline (2018) [34] & II & N/A & N/A & 26 & $\begin{array}{l}(56- \\
85)\end{array}$ & $\begin{array}{l}\text { (+pembrolizumab-PD- } \\
1 \text { inhibitor in 13) }\end{array}$ & $<2$ & cancer & Radiation, chemo, abiraterone, enzalutamide & $\begin{array}{l}19 \%, 8=19 \%, 9 \\
=54 \%\end{array}$ & N/A & 165) \\
\hline $\begin{array}{l}\text { Gross et al. } \\
\text { (2017) [11] }\end{array}$ & lb & NCT00574769 & $\begin{array}{l}12 \text { cycles }+ \\
\text { maintenance }\end{array}$ & 43 & $\begin{array}{l}65 \\
(50- \\
79)\end{array}$ & $\begin{array}{l}\text { Docetaxel, } \\
\text { bevacizumab, and } \\
\text { everolimus }\end{array}$ & N/A & $\begin{array}{l}\text { Bone }=88 \%, \\
\text { nodes }=44 \%, \\
\text { viscera }=19 \%\end{array}$ & $\begin{array}{l}\text { Abiraterone }=26 \% \text {, orteronel }=7 \% \text {, } \\
\text { enzalutamide }=5 \%\end{array}$ & $\mathrm{~N} / \mathrm{A}$ & $\begin{array}{l}\text { Bone } \\
\text { metastasis = } \\
88 \%\end{array}$ & $\begin{array}{l}76.6(0- \\
1847)\end{array}$ \\
\hline $\begin{array}{l}\text { Cathomas et } \\
\text { al. (2012) [15] }\end{array}$ & " & NCT00728663 & 25.4 months & 38 & $\begin{array}{l}68 \\
(45- \\
82)\end{array}$ & $\begin{array}{l}\text { Docetaxel + } \\
\text { cetuximab (EGFR } \\
\text { inhibitor, } 400 \mathrm{mg} / \mathrm{m} 2 \text { ) }\end{array}$ & N/A & $\begin{array}{l}\text { Bone }=89 \% \\
\text { node }=63 \% \\
\text { visceral }=34 \%\end{array}$ & $\begin{array}{l}1 \text { regimen }=65 \%, 2 \text { regimens }=26 \%, 3 \\
\text { regimens }=9 \% \text { (docetaxel regimens) }\end{array}$ & N/A & $\begin{array}{l}\text { Bone } \\
\text { metastasis = } \\
89 \%\end{array}$ & $\begin{array}{l}212 \\
\mathrm{ng} / \mathrm{ml} \\
(4.4- \\
8,898)\end{array}$ \\
\hline $\begin{array}{l}\text { Batra et al. } \\
\text { (2020) [35] }\end{array}$ & 1 & NCT00916123 & N/A & 15 & $\begin{array}{l}69 \\
(49- \\
80)\end{array}$ & $\begin{array}{l}\text { Docetaxel + J591 } \\
(177 L \text { L-J591) }\end{array}$ & $\begin{array}{l}0=40 \%, \\
1= \\
53.3 \%, 2 \\
=6.7 \%\end{array}$ & $\begin{array}{l}\text { Bone }=93.3 \% \\
\text { node }=60 \% \\
\text { lung }=6.7 \%\end{array}$ & $\begin{array}{l}\text { Primary radiotherapy }=40 \% \text {, salvage } \\
\text { radiotherapy }=13.3 \%, \text { prostatectomy }= \\
46.7 \%\end{array}$ & $\begin{array}{l}6=13.3 \%, 7= \\
40 \%, 8-10=40 \%\end{array}$ & $\begin{array}{l}\text { Bone } \\
\text { metastasis = } \\
93.3 \%\end{array}$ & $\begin{array}{l}84.32 \\
\mathrm{ng} / \mathrm{ml} \\
(17.2- \\
776)\end{array}$ \\
\hline $\begin{array}{l}\text { Madan et al. } \\
\text { (2016) [36] }\end{array}$ & " & NCT00942578 & 47.5 months & 63 & $\begin{array}{l}65.6 \\
(51- \\
82)\end{array}$ & $\begin{array}{l}\text { enalidomide with } \\
\text { bevacizumab, } \\
\text { docetaxel, and } \\
\text { prednisone }\end{array}$ & $\begin{array}{l}0=10,1 \\
=50,2= \\
3\end{array}$ & $\begin{array}{l}\text { Bone }=24, \\
\text { bone }+ \text { nodes }= \\
27, \text { bone }+ \\
\text { visceral }=7\end{array}$ & N/A & $\begin{array}{l}\leq 6=4,7=15,8 \\
=15,9=23,10 \\
=6\end{array}$ & Bone $=24$ & $\begin{array}{l}90.36 \\
(0.14-3 \\
520)\end{array}$ \\
\hline \multirow{2}{*}{$\begin{array}{l}\text { Slovin et al. } \\
\text { (2013) [37] }\end{array}$} & \multirow{2}{*}{ I/II } & \multirow{2}{*}{ NCT00323882 } & \multirow{2}{*}{$\mathrm{N} / \mathrm{A}$} & 16 & $\begin{array}{l}65 \\
(53- \\
76)\end{array}$ & $\begin{array}{l}\text { Ipilimumab (anti- } \\
\text { CTLA-4, } 10 \text { mg/kg) }\end{array}$ & $\begin{array}{l}0=10,1 \\
=6,2= \\
0\end{array}$ & $\begin{array}{l}\text { Metastatic } \\
\text { cancer }\end{array}$ & $6(38 \%)$ & $\mathrm{N} / \mathrm{A}$ & $2.5(1-12)$ & $\begin{array}{l}132(13- \\
2581)\end{array}$ \\
\hline & & & & 34 & $\begin{array}{l}66 \\
(50- \\
83)\end{array}$ & $\begin{array}{l}\text { Ipilimumab = } 10 \\
\mathrm{mg} / \mathrm{kg}+\mathrm{XRT}\end{array}$ & $\begin{array}{l}0=9,1 \\
=22,2= \\
0\end{array}$ & $\begin{array}{l}\text { Metastatic } \\
\text { cancer }\end{array}$ & $21(62 \%)$ & N/A & $8(1-15)$ & $\begin{array}{l}120(8- \\
1314)\end{array}$ \\
\hline $\begin{array}{l}\text { Barata et al. } \\
\text { (2019) [38] }\end{array}$ & I/II & NCT01083368 & N/A & 21 & $\begin{array}{l}64 \\
(53- \\
82)\end{array}$ & $\begin{array}{l}\text { Temsirolimus and } \\
\text { bevacizumab }\end{array}$ & $\begin{array}{l}0=19 \% \\
1=62 \% \\
2=14 \%\end{array}$ & $\begin{array}{l}\text { Metastatic } \\
\text { cancer }\end{array}$ & $\begin{array}{l}\text { Docetaxel }=86 \% \text {, mitoxantrone }=29 \%, \\
\text { ketoconazole }=24 \% \text {, cabazitaxel }=10 \%, \\
\text { gemcitabine }=10 \%\end{array}$ & $\begin{array}{l}<7=33 \%,>=8= \\
43 \%\end{array}$ & $21(100 \%)$ & $\begin{array}{l}205.3 \\
(11.1- \\
1801.0)\end{array}$ \\
\hline $\begin{array}{l}\text { Autio et al. } \\
\text { (2020) [39] }\end{array}$ & 1 & NCT02265536 & N/A & 12 & $\begin{array}{l}58- \\
84\end{array}$ & LY3022855 & $\begin{array}{l}0=33 \% \\
1= \\
58.3 \%, 2 \\
=8 \%\end{array}$ & $\begin{array}{l}\text { Metastatic } \\
\text { cancer }\end{array}$ & $\begin{array}{l}\text { Chemotherapy }=42 \% \text { Abiraterone } \\
\text { acetate/enzalutamide }=100 \%\end{array}$ & N/A & 10/12 (83\%) & N/A \\
\hline $\begin{array}{l}\text { Di Lorenzo et } \\
\text { al. (2008) [40] }\end{array}$ & " & N/A & N/A & 20 & $\begin{array}{l}66 \\
(49- \\
73)\end{array}$ & $\begin{array}{l}\text { Bevacizumab + } \\
\text { docetaxel }\end{array}$ & N/A & $\begin{array}{l}\text { Metastatic } \\
\text { cancer }\end{array}$ & $\begin{array}{l}\text { Docetaxel }=100 \%, \text { mitoxantrone = } 100 \%, \\
\text { vinorelbine }=65 \%\end{array}$ & $<7=8,>7=12$ & $\begin{array}{l}\text { Bone } \\
\text { metastasis = } \\
100 \%\end{array}$ & 260 \\
\hline $\begin{array}{l}\text { Graff et al. } \\
\text { (2020) [42] }\end{array}$ & "I & NCT02312557 & 37 months & 28 & $\begin{array}{l}72 \\
(61- \\
90)\end{array}$ & $\begin{array}{l}\text { Pembrolizumab (anti- } \\
\text { PD-1, 200mg) }+ \\
\text { enzalutamide }\end{array}$ & $\begin{array}{l}0=39 \% \\
1=61 \%\end{array}$ & $\begin{array}{l}\text { Metastatic } \\
\text { cancer }\end{array}$ & $\begin{array}{l}\text { Docetaxel }=4, \text { abiraterone }=10, \\
\text { enzalutamide }=28\end{array}$ & $\begin{array}{l}<7=1,7=9,>7 \\
=14\end{array}$ & $\begin{array}{l}\text { Bone only = } \\
13 \text {, bone and } \\
\text { lymph nodes } \\
=9\end{array}$ & $\begin{array}{l}26.61 \\
\mathrm{ng} / \mathrm{ml} \\
(3.03- \\
2502.75)\end{array}$ \\
\hline $\begin{array}{l}\text { Francini et al. } \\
\text { (2011) [43] }\end{array}$ & "I & N/A & 11.3 months & 43 & $\begin{array}{l}74 \\
(58- \\
82)\end{array}$ & $\begin{array}{l}\text { Docetaxel + } \\
\text { bevacizumab + } \\
\text { prednisone }\end{array}$ & $\begin{array}{l}0= \\
20.9 \% \\
1-2= \\
79 \%\end{array}$ & $\begin{array}{l}\text { Metastatic } \\
\text { cancer }\end{array}$ & $\begin{array}{l}\text { W-epirubicin + w-docetaxel = } 213-\mathrm{w}_{\mathrm{t}} \\
\text { docetaxel }+ \text { prednisone }=15, \mathrm{w} \text {-docetaxel }+ \\
\text { prednisone }=7\end{array}$ & N/A & N/A & $\begin{array}{l}78(47- \\
374)\end{array}$ \\
\hline $\begin{array}{l}\text { Ning et al. } \\
\text { (2010) [44] }\end{array}$ & " & N/A & 34 months & 60 & $\begin{array}{l}66 \\
(44- \\
79)\end{array}$ & $\begin{array}{l}\text { Docetaxel, } \\
\text { bevacizumab, } \\
\text { thalidomide, } \\
\text { prednisone }\end{array}$ & $\begin{array}{l}0=13 \% \\
1=80 \% \\
2=7 \%\end{array}$ & $\begin{array}{l}\text { Metastatic } \\
\text { cancer }\end{array}$ & N/A & $\begin{array}{l}<7=20(33 \%), \\
>8=39(65 \%)\end{array}$ & N/A & $\begin{array}{l}99(0.9- \\
4,399)\end{array}$ \\
\hline $\begin{array}{l}\text { Hudes et al. } \\
\text { (2013) [41] }\end{array}$ & 1 & N/A & N/A & 39 & $\begin{array}{l}66 \\
(43, \\
82)\end{array}$ & $\begin{array}{l}\text { Docetaxel } 75 \mathrm{mg} / \mathrm{m} 2+ \\
\text { siltuximab (anti-lL-6, } \\
6-12 \mathrm{mg} / \mathrm{kg} \text { ) }\end{array}$ & N/A & $\begin{array}{l}\text { Metastatic } \\
\text { cancer }\end{array}$ & N/A & $8(5,10)$ & N/A & $\begin{array}{l}57(12, \\
1430)\end{array}$ \\
\hline \multirow{2}{*}{$\begin{array}{l}\text { Sharma et al. } \\
\text { (2020) [16] }\end{array}$} & \multirow{2}{*}{ "I } & \multirow{2}{*}{ NCT02985957 } & 11.9 months & 45 & $\begin{array}{l}69 \\
(48- \\
85)\end{array}$ & $\begin{array}{l}\text { Nivolumab (anti-PD-1, } \\
1 \mathrm{mg} / \mathrm{kg} \text { ) + ipilimumab } \\
\text { (anti-CTLA-4, } 3 \\
\mathrm{mg} / \mathrm{kg} \text { ) }\end{array}$ & $\begin{array}{l}0=26 \\
(57.8 \%), \\
1=19 \\
(42.2 \%)\end{array}$ & $\begin{array}{l}\mathrm{M} 0=28 \\
(62.2 \%), \mathrm{Ml}= \\
15(33.3 \%)\end{array}$ & $\begin{array}{l}\text { Abiraterone }=66.7 \%, \text { enzalutamide }= \\
57.8 \% \text {, bicalutamide }=55.6 \%, \text { leuprolide }= \\
60 \%, \text { docetaxel }=11.1 \%\end{array}$ & $\begin{array}{l}<7=35.5 \%,>7= \\
60 \%\end{array}$ & $\begin{array}{l}0=20 \%,<4 \\
=13.3 \%,>4 \\
=66.7 \%\end{array}$ & $\begin{array}{l}59.5 \\
\mathrm{ng} / \mathrm{ml} \\
(93.3- \\
1045)\end{array}$ \\
\hline & & & 13.5 months & 45 & $\begin{array}{l}65 \\
(46- \\
84)\end{array}$ & $\begin{array}{l}\text { Nivolumab } 1 \mathrm{mg} / \mathrm{kg}+ \\
\text { ipilimumab (3 mg/kg) }\end{array}$ & $\begin{array}{l}(55.6) 1 \\
=20 \\
(44.4 \%)\end{array}$ & $\begin{array}{l}\mathrm{MO}=22 \\
(48.9 \%), \mathrm{MI}= \\
20(44.4 \%)\end{array}$ & $\begin{array}{l}\text { Abiraterone }=71.1 \% \text {, enzalutamide }= \\
62.2 \%, \text { bicalutamide }=64.4 \% \text {, leuprolide }= \\
53.3 \% \text {, docetaxel }=86.7 \% \text {, cabazitaxel }= \\
46.7 \%\end{array}$ & $\begin{array}{l}7 \text { or less }= \\
42.2 \%, 8 \text { or more } \\
=51.1 \%\end{array}$ & $\begin{array}{l}0=6.7 \%,<4 \\
=2.2 \%,>4= \\
91.1 \%\end{array}$ & $\begin{array}{l}158.9 \\
\mathrm{ng} / \mathrm{ml} \\
(1.8- \\
1348.7)\end{array}$ \\
\hline
\end{tabular}




\section{Cureus}

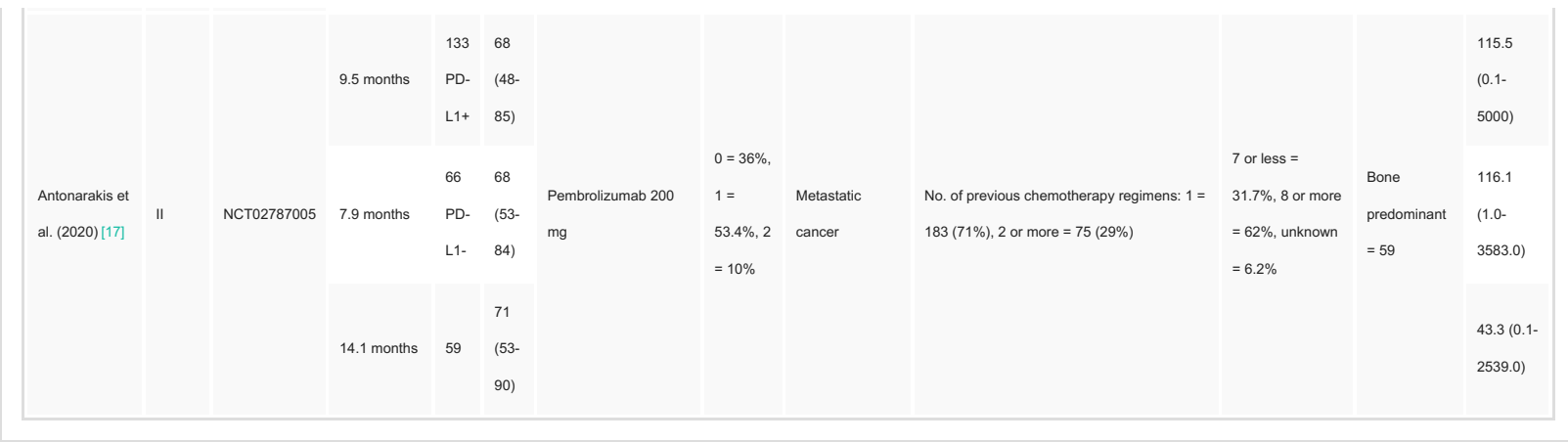

\section{TABLE 1: Baseline characteristics of trials.}

NCT = National Clinical Trial ECOG = Eastern Cooperative Oncology Group; PSA = prostate-specific antigen; CTLA-4 = cytotoxic T-lymphocyteassociated antigen-4; RANKL = receptor activator of nuclear factor kappa-B ligand; $M=$ mitoxantrone; $P=$ prednisone; $X R T=$ radiation therapy; $S O C=$ standard of care; VEGFR = vascular endothelial growth factor receptor; EGFR = epidermal growth factor receptor; PD-1 = programmed cell death protein $1 ;$ IL-6 = interleukin 6; IGF = insulin-like growth factor; HER2 = human epidermal growth factor receptor 2; VEGF-A = vascular endothelial growth factor A.

\section{Efficacy}

In RCTs with ipilimumab, denosumab, bevacizumab, and abituzumab ( $\mathrm{N}=4,063)$, pooled hazard ratio (HR) of PFS was $0.77\left(95 \% \mathrm{CI}=0.69-0.87, \mathrm{I}^{2}=53\right)$ in favor of monoclonal antibodies as compared to placebo. HR of PFS for trial on intetumumab $(\mathrm{N}=131)$ was $1.73(95 \% \mathrm{CI}=1.11-2.69)$ in favor of placebo as compared to monoclonal antibodies (Figure $3 A$ ).

In RCTs with ipilimumab and bevacizumab $(\mathrm{N}=2,254)$, the risk ratio (RR) of $\geqslant 50 \%$ decline in PSA was 1.99 (95\% CI $=0.97-4.08, \mathrm{I}^{2}=53 \%$ ) in favor of monoclonal antibodies as compared to placebo. While in the RCT with intetumumab, RR of $\geqslant 50 \%$ decline in PSA was $0.62(95 \% \mathrm{CI}=0.44-0.87)$ in favor of placebo as compared to monoclonal antibodies (Figure 3B).

In RCTs with ipilimumab, denosumab, bevacizumab, and intetumumab ( $\mathrm{N}=4014)$, HR of overall survival was similar in monoclonal antibodies groups vs. placebo, i.e., 0.98 (95\% CI = 0.86-1.11, $\mathrm{I}^{2}=40 \%$ ) (Figure $3 \mathrm{C}$ ). 


\section{Cureus}

A

\begin{tabular}{|c|c|}
\hline Study & seTE \\
\hline \multicolumn{2}{|l|}{ main studies } \\
\hline Beer et al. 2017 (Ipilimumab) & $\begin{array}{lll}-0.40 & 0.0988\end{array}$ \\
\hline Smith et al. 2012 (Denosumab) & -0.120 .0684 \\
\hline Kwon et al. 2014 (Ipilimumab) & -0.360 .0755 \\
\hline Kelly et al. 2012 (Bevacizumab) & -0.220 .0633 \\
\hline Hussain et al. 2016 (Abituzumab) & -0.160 .1938 \\
\hline \multirow{2}{*}{\multicolumn{2}{|c|}{$\begin{array}{l}\text { Random effects model } \\
\text { Heterogeneity: } I^{2}=53 \%, \tau^{2}=0.0078, p=0.08\end{array}$}} \\
\hline & \\
\hline \multicolumn{2}{|l|}{ Outlier } \\
\hline \multirow{3}{*}{\multicolumn{2}{|c|}{$\begin{array}{l}\text { Heidenreich et al. } 2013 \text { (Intetumumab) } \\
\text { Random effects model } \\
\text { Heterogeneity: not applicable }\end{array}$}} \\
\hline & \\
\hline & \\
\hline \multicolumn{2}{|c|}{$\begin{array}{l}\text { Random effects model } \\
\text { Heterogeneity } I^{2}=76 \% \tau^{2}=0.0261, p<0.01\end{array}$} \\
\hline \multicolumn{2}{|l|}{ Residual heterogeneity: $I^{2}=53 \%, p=0.08$} \\
\hline
\end{tabular}

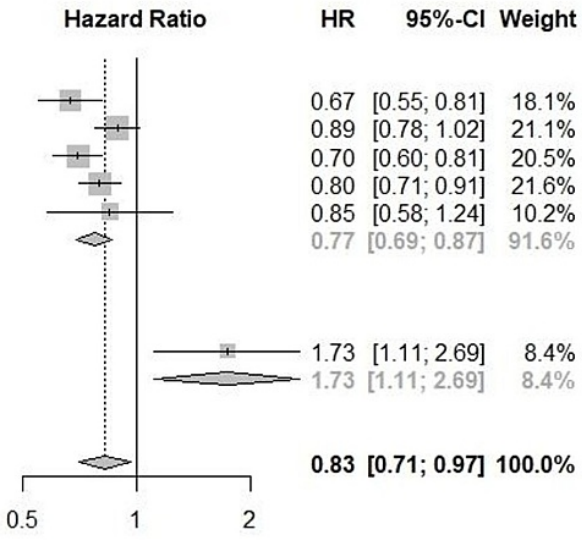

B

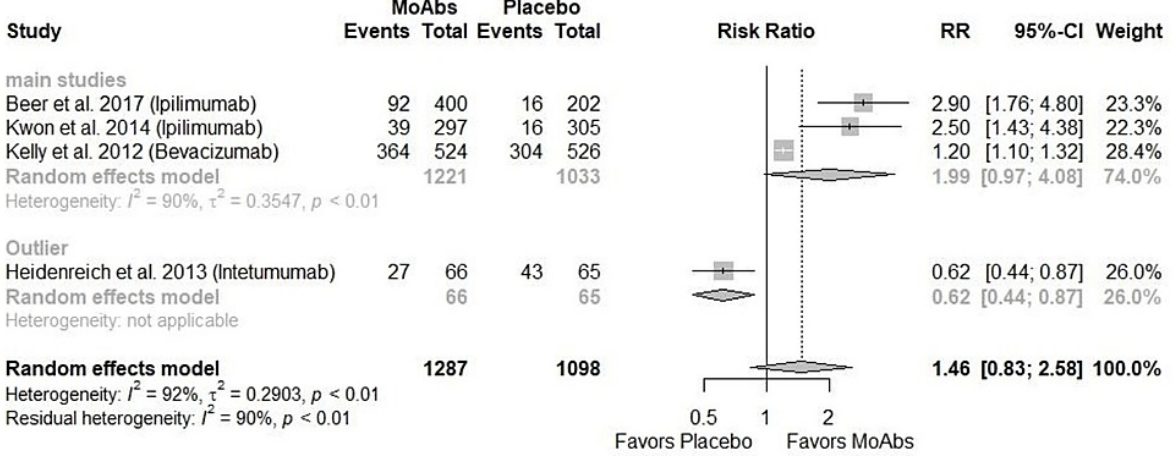

C

\begin{tabular}{|c|c|c|c|c|c|}
\hline Study & TE seTE & Hazard Ratio & HR & $95 \%-\mathrm{Cl}$ & Weight \\
\hline Beer et al. 2017 (Ipilimumab) & 0.100 .1166 & & 1.11 & {$[0.88 ; 1.40]$} & $19.1 \%$ \\
\hline Smith et al. 2012 (Denosumab) & 0.010 .0880 & & 1.01 & {$[0.85 ; 1.20]$} & $26.3 \%$ \\
\hline Heidenreich et al. 2013 (Intetumumab) & 0.390 .2772 & & 1.47 & {$[0.85 ; 2.53]$} & \\
\hline Kwon et al. 2014 (Ipilimumab) & $\begin{array}{ll}-0.16 & 0.0838\end{array}$ & 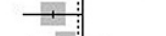 & 0.85 & {$[0.72 ; 1.00]$} & 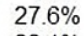 \\
\hline Kelly et al. 2012 (Bevacizumab) & -0.090 .1034 & 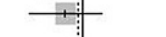 & 0.91 & {$[0.74 ; 1.11]$} & 22.1 \\
\hline \multirow{2}{*}{\multicolumn{2}{|c|}{$\begin{array}{l}\text { Random effects model } \\
\text { Heterogeneity: } I^{2}=40 \%, \tau^{2}=0.0078, p=0.16\end{array}$}} & & \multirow{2}{*}{\multicolumn{3}{|c|}{$0.98[0.86 ; 1.11] 100.0 \%$}} \\
\hline & & & & & \\
\hline
\end{tabular}

FIGURE 3: Comparison of efficacy in monoclonal antibodies vs. placebo.

(A) Hazard ratio of progression-free survival. (B) Risk ratio of $\geq 50 \%$ decline in prostate-specific antigen (PSA). (C) Hazard ratio of overall survival [24-30].

MoAbs = monoclonal antibodies; TE = treatment effect; seTE: standard error of treatment effect.

In RCT with denosumab ( $\mathrm{N}=1432$ ), HRs of bone metastasis-free survival and first bone metastasis were statistically significant in favor of denosumab. HRs of bone metastasis-free survival and first bone metastasis were $0.85(95 \% \mathrm{CI}=0.73-0.98)$ and $0.84(95 \% \mathrm{CI}=0.71-0.98)$, respectively.

\section{Safety}

In RCTs, RRs of any $\geqslant$ grade 3 toxicity were $1.41\left(\mathrm{CI}=1.10-1.82, \mathrm{I}^{2}=92 \%\right)$ in favor of placebo as compared to monoclonal antibodies. RRs of $\geqslant$ grade 3 adverse events, i.e., vomiting, rash, pancreatitis, neutropenia, hypertension, hepatitis, fatigue, diarrhea, colitis, and anemia, were $5.30\left(95 \% \mathrm{CI}=0.87-32.36, \mathrm{I}^{2}=0\right), 7.50$ $\left(95 \% \mathrm{CI}=0.94-59.46, \mathrm{I}^{2}=0\right), 9.21(95 \% \mathrm{CI}=4.27-19.85), 1.01\left(95 \% \mathrm{CI}=0.58-1.74, \mathrm{I}^{2}=63.5 \%\right), 3.98(95 \% \mathrm{CI}=$ 


\section{Cureus}

$\left.1.23-12.84, \mathrm{I}^{2}=19.2 \%\right), 5.02\left(95 \% \mathrm{CI}=0.58-42.95, \mathrm{I}^{2}=0\right), 1.44\left(95 \% \mathrm{CI}=1.00-2.07, \mathrm{I}^{2}=22.8 \%\right), 4.42(95 \% \mathrm{CI}$ $\left.=0.25-75.69, \mathrm{I}^{2}=81.1 \%\right), 2.82\left(95 \% \mathrm{CI}=0.01-550.00, \mathrm{I}^{2}=84.6 \%\right)$, and $1.28\left(95 \% \mathrm{CI}=0.79-2.08, \mathrm{I}^{2}=8.3 \%\right)$, respectively. Denosumab increased the incidence of $\geqslant$ grade 3 osteonecrosis of jaw in RCT 33/720 vs. 0/705 (Figure 4).

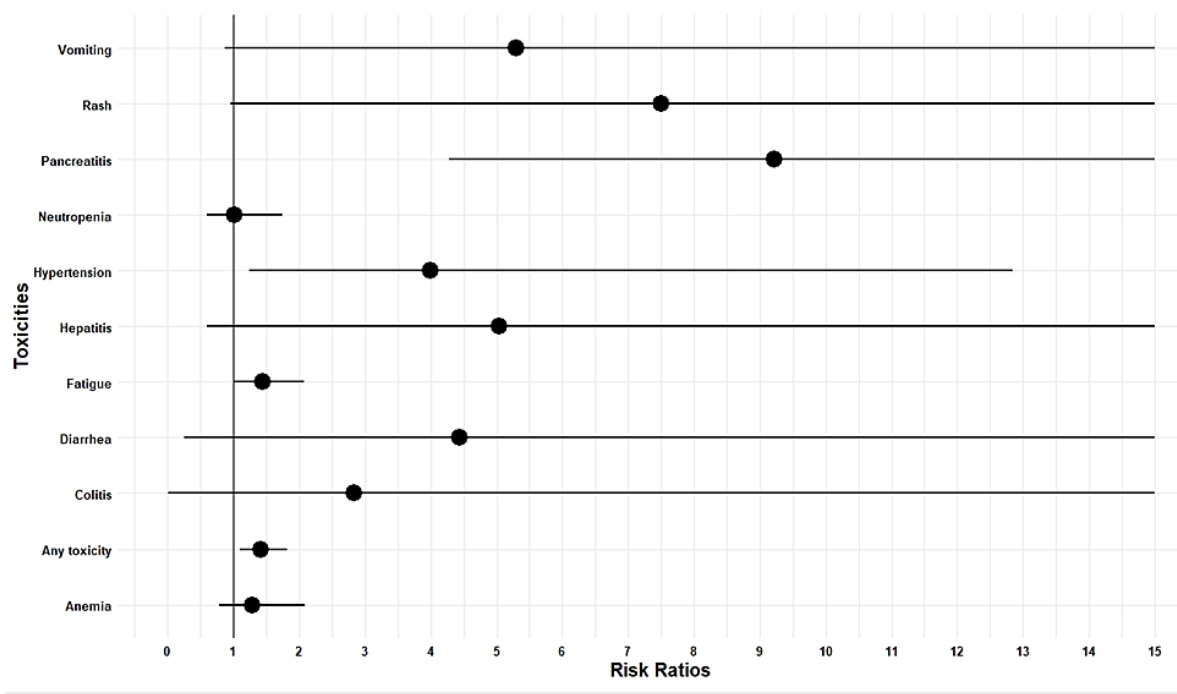

FIGURE 4: Plot of the risk ratio of $\geq$ grade 3 adverse events.

\section{Denosumab vs. zoledronic acid}

Fizazi et al. (2011) [26] compared denosumab vs. zoledronic acid for the treatment of CRPC $(N=1,904)$. HR of the first skeletal-related adverse event was $0.82(95 \% \mathrm{CI}=0.71-0.95)$ in favor of denosumab as compared to zoledronic acid. The incidence of total skeletal-related events was $36 \%$ in the denosumab group vs. $41 \%$ in the zoledronic acid group. Radiation to bone was used in $19 \%$ of the people in the denosumab group vs. $21 \%$ in the zoledronic acid group. The incidence of adverse events was $97 \%$ each in both groups. Greater than or equal to grade 3 adverse events were $72 \%$ and $66 \%$ in denosumab and zoledronic acid groups, respectively. Osteonecrosis of the jaw was $1 \%$ in the zoledronic acid group vs. $2 \%$ in the denosumab group. Discontinuation of treatment due to adverse events was reported in $15 \%$ of participants in the zoledronic acid group and $17 \%$ in the denosumab group.

\section{Cixutumumab vs. ramucirumab}

Hussain et al. (2015) [30] compared cixutumumab vs. ramucirumab ( $N=132)$. The median time to radiographic disease progression was 7.5 months $(95 \% \mathrm{CI}=4.8-10.1)$ for patients on cixutumumab while it was 10.2 months $(95 \% \mathrm{CI}=7.5-12.6)$ for patients on ramucirumab. Median OS was 10.8 months $(95 \% \mathrm{CI}=$ 6.5-13.0) for patients on cixutumumab while it was 13.0 months $(95 \% \mathrm{CI}=9.5-16.0)$ for patients on ramucirumab. Decline $>50 \%$ in PSA occurred in $18.5 \%$ of patients in the cixutumumab group and $21.4 \%$ of patients in the ramucirumab group. Among $\geqslant$ grade 3 adverse events, fatigue, diarrhea, dehydration, hypertension, neutropenia, and anemia were reported in $16.7 \%$ vs. $7.6 \%, 7.6 \%$ vs. $1.5 \%, 6.1 \%$ vs. $1.5 \%, 1.5 \%$ vs. $9.1 \%, 31.9 \%$ vs. $31.8 \%$, and $3 \%$ vs. $10.6 \%$ of patients, respectively, in cixutumumab vs. ramucirumab groups.

\section{Single-arm comparison of monoclonal antibody regimens}

Ipilimumab, cixutumumab, ramucirumab, anti-CD3 $\mathrm{x}$ anti-HER2 bispecific antibody, and pembrolizumab were used as monotherapy in clinical trials ( $N=1,129)[17,24,28,30,31]$. Pooled incidences of OR and $>50 \%$ decline in PSA were $8 \%\left(95 \% \mathrm{CI}=0.03-0.22, \mathrm{I}^{2}=89 \%\right)$ and $15 \%\left(95 \% \mathrm{CI}=0.1-0.23, \mathrm{I}^{2}=83 \%\right)$, respectively. Individual study results and pooled results are given in Figure 5. Median OS and PFS were 7.4-19.6 months and 2.1 months, respectively (Table 2). 


\section{Cureus}

A

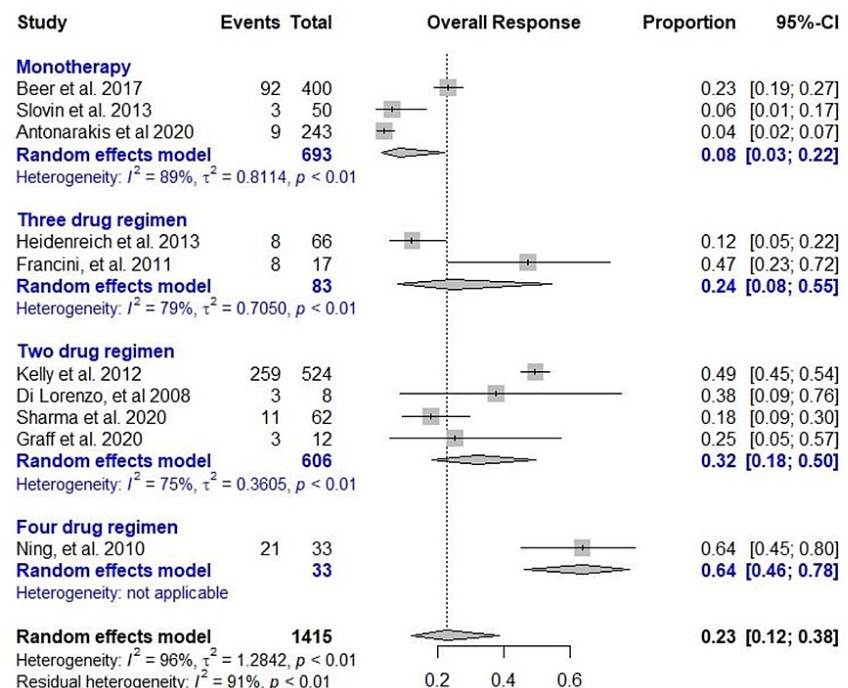

B

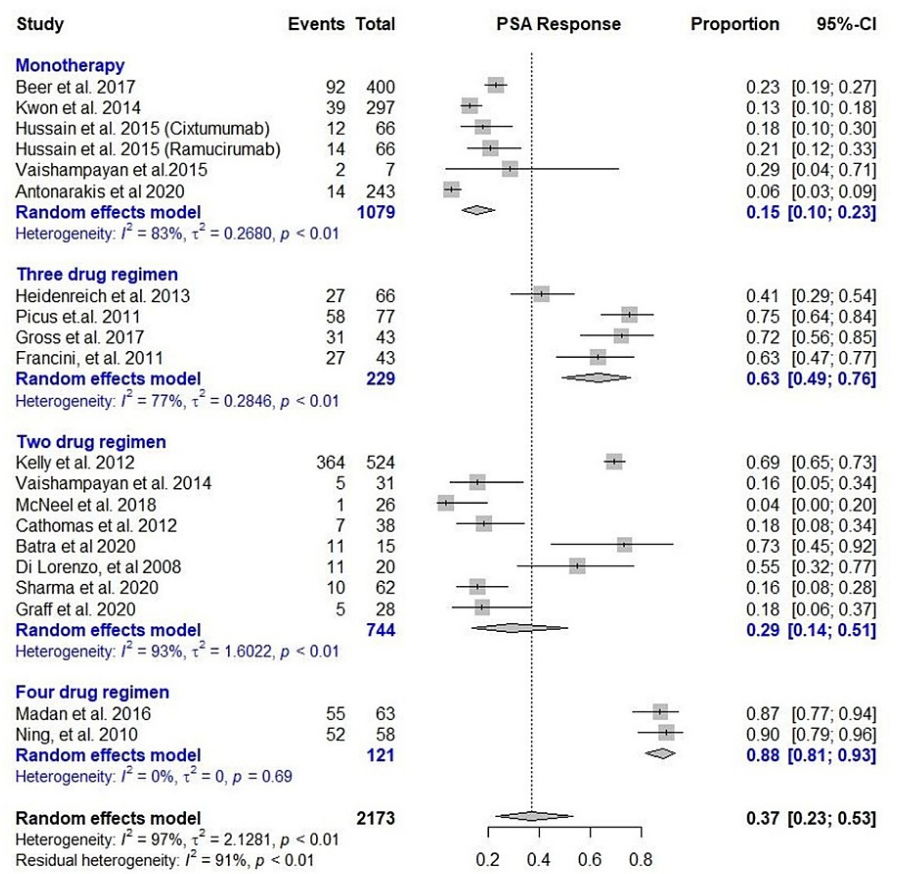

FIGURE 5: Meta-analysis of efficacy in single arms.

(A) Pooled overall response. (B) Pooled $>50 \%$ prostate-specific antigen (PSA) response [10,17,24-35]. 


\section{Cureus}

\begin{tabular}{|c|c|c|c|c|c|c|c|c|c|c|c|c|}
\hline Author & Median PFS (months) & Median OS (months) & $\begin{array}{l}\text { Any } \geq \\
\text { grade } 3\end{array}$ & Diarrhea & Hypertension & Anemia & Neutropenia/lymphopenia & Colitis & Hepatitis & Fatigue & Rash & Vomiting \\
\hline \multicolumn{13}{|l|}{ Monotherapy } \\
\hline $\begin{array}{l}\text { Vaishampayan et al. } \\
\text { (2015) [31] }\end{array}$ & N/A & N/A & $5 / 7$ & N/A & N/A & N/A & N/A & N/A & N/A & N/A & N/A & N/A \\
\hline $\begin{array}{l}\text { Antonarakis et al. (2020) } \\
\text { [17] }\end{array}$ & $2.1(2.1-2.2)$ & $9.6(7.9-12.2)$ & $27(10 \%)$ & $2(<1 \%)$ & N/A & $2(<1 \%)$ & N/A & $3(1 \%)$ & $1(<1 \%)$ & $3(1 \%)$ & 0 & 0 \\
\hline Slovin et al. (2013) [17] & N/A & $17.4(11.5-24.7)$ & N/A & $4(8)$ & N/A & N/A & N/A & $8(16)$ & $4(8)$ & $3(6)$ & $\begin{array}{l}16 \\
(32 \%)\end{array}$ & $3(6 \%)$ \\
\hline Autio et al. (2020) [39] & N/A & N/A & 3 & N/A & N/A & N/A & N/A & N/A & N/A & 2 & N/A & 0 \\
\hline \multicolumn{13}{|l|}{ Two-drug regimens } \\
\hline $\begin{array}{l}\text { Vaishampayan et al. } \\
\text { (2014) [32] }\end{array}$ & $7.0(4.7-8.5)$ & $11.2(9.1-16.4)$ & N/A & $2 / 30$ & $3 / 30$ & $7 / 30$ & $9 / 30$ & N/A & N/A & $1 / 30$ & N/A & N/A \\
\hline McNeel et al. (2018) [34] & N/A & N/A & N/A & $1 / 26$ & N/A & N/A & N/A & N/A & $1 / 26$ & $1 / 26$ & N/A & N/A \\
\hline $\begin{array}{l}\text { Cathomas et al. (2012) } \\
\text { [15] }\end{array}$ & $2.8(2.4-3.2)$ & $13.3(7.3-15.4)$ & N/A & $1(3 \%)$ & N/A & $1(3 \%)$ & $3(8 \%)$ & N/A & N/A & $4(11 \%)$ & $\begin{array}{l}2 \\
(5 \%)\end{array}$ & N/A \\
\hline Batra et al. (2020) [35] & N/A & 18.4 (16.13-NR) & N/A & N/A & N/A & N/A & $11(73.3 \%)$ & N/A & N/A & $\begin{array}{l}1 \\
(6.66 \%)\end{array}$ & N/A & N/A \\
\hline $\begin{array}{l}\text { Di Lorenzo et al. (2008) } \\
\text { [40] }\end{array}$ & $4(2-6)$ & $9(4-12.5)$ & $\begin{array}{l}11 / 20 \\
(55 \%)\end{array}$ & N/A & N/A & $1 / 20(5 \%)$ & $4(20 \%)$ & N/A & N/A & N/A & N/A & $2(10 \%)$ \\
\hline Sharma et al. (2020) [16] & $\begin{array}{l}5.5(3.5-7.1) \text { and } 3.8 \\
(2.1-5.1)\end{array}$ & $\begin{array}{l}19 \text { (11.5-NE) and } 15.2 \\
(8.4-\mathrm{NE})\end{array}$ & $43 / 85$ & 8 & $\mathrm{~N} / \mathrm{A}$ & N/A & N/A & N/A & N/A & N/A & N/A & N/A \\
\hline Graff et al. (2020) [42] & $3.8(2.8-9.9)$ & $22.2(14.7-28.4)$ & $\begin{array}{l}19 / 28 \\
(68 \%)\end{array}$ & N/A & $3(10.7 \%)$ & $1(3.5 \%)$ & N/A & $\begin{array}{l}2 \\
(7.1 \%)\end{array}$ & N/A & $\begin{array}{l}1 \\
(3.5 \%)\end{array}$ & N/A & N/A \\
\hline Barata et al. (2019) [38] & N/A & N/A & $9(43 \%)$ & N/A & N/A & $N / A$ & N/A & N/A & N/A & $5(24 \%)$ & N/A & $1(5 \%)$ \\
\hline Hudes et al. (2013) [41] & N/A & N/A & $33 / 37$ & 0 & N/A & 1 & 27/37 (73\%) & N/A & N/A & N/A & N/A & N/A \\
\hline \multicolumn{13}{|l|}{ Three-drug regimens } \\
\hline Francini et al. (2011) [43] & N/A & N/A & $\begin{array}{l}16 / 43 \\
(37.2 \%)\end{array}$ & N/A & N/A & $6(13.9 \%)$ & $8(18.6 \%)$ & N/A & N/A & $\begin{array}{l}2 \\
(4.6 \%)\end{array}$ & N/A & N/A \\
\hline Picus et al. (2011) [33] & $9.2(7.5-10.9)$ & $24(20.3-26.5$ & N/A & N/A & $4 / 77(5 \%)$ & N/A & $53 / 77(69 \%)$ & N/A & N/A & $\begin{array}{l}19 \\
(24 \%)\end{array}$ & N/A & N/A \\
\hline Gross et al. (2017) [11] & $8.9(7.4-10.6)$ & $21.9(18.4-30.3)$ & N/A & N/A & $8(19 \%)$ & N/A & $12(28 \%)$ & N/A & N/A & $3(7 \%)$ & N/A & N/A \\
\hline \multicolumn{13}{|l|}{ Four-drug regimens } \\
\hline Ning et al. (2010) [44] & 18.3 & 28.2 & N/A & $\begin{array}{l}2 / 60 \\
(3.33 \%)\end{array}$ & $7 / 60(11.6 \%)$ & $\begin{array}{l}8 / 60 \\
(13.3 \%)\end{array}$ & $60 / 60(100 \%)$ & N/A & N/A & $\begin{array}{l}2 / 60 \\
(3.33 \%)\end{array}$ & N/A & N/A \\
\hline Madan et al. (2016) [36] & 18.2 & 24.6 & N/A & $6(10 \%)$ & N/A & $20(32 \%)$ & 61 & N/A & N/A & $6(11 \%)$ & N/A & N/A \\
\hline
\end{tabular}

TABLE 2: Survival rates and $\geq$ grade 3 adverse events in early phase trials.

PFS = progression-free survival; OS = overall survival

Bevacizumab + docetaxel, bevacizumab + satraplatin, anti-tumor vaccine + pembrolizumab, docetaxel + cetuximab, docetaxel + J591, bevacizumab + docetaxel, nivolumab + ipilimumab, and pembrolizumab + enzalutamide were two drug combination regimens used in clinical trials to treat CRPC $(\mathrm{N}=744)$ $[15,16,32,34,40,41]$. Pooled incidences of OR and $>50 \%$ decline in PSA were $32 \%\left(95 \% \mathrm{CI}=0.18-0.50, \mathrm{I}^{2}=\right.$ $75 \%)$ and $29 \%\left(95 \% \mathrm{CI}=0.14-0.51, \mathrm{I}^{2}=93 \%\right)$, respectively (Figure 5). Median OS and PFS were 9-19 months and 2.8-7 months, respectively (Table 2). 


\section{Cureus}

Intetumumab + docetaxel + prednisone, estramustine + docetaxel + bevacizumab, docetaxel + bevacizumab + everolimus, and docetaxel + bevacizumab + prednisone were the three-drug regimens used in clinical trials $(\mathrm{N}=229)[10,27,33,40,43]$. Pooled incidences of OR and $>50 \%$ decline in PSA were $24 \%(95 \% \mathrm{CI}=0.08-0.55$, $\left.\mathrm{I}^{2}=79 \%\right)$ and $63 \%\left(95 \% \mathrm{CI}=0.49-0.76, \mathrm{I}^{2}=77 \%\right)$, respectively (Figure 5). Median OS and PFS were 21.9-24 months and 9.2-8.9 months, respectively (Table 2).

Lenalidomide + bevacizumab + docetaxel + prednisone and docetaxel + bevacizumab + thalidomide + prednisone were the four-drug regimens used in clinical trials $(\mathrm{N}=121)[36,44]$. Pooled incidences of OR and $>50 \%$ decline in PSA were 64\% (95\% CI $\left.=0.46-0.78, \mathrm{I}^{2}=0 \%\right)$ and $88 \%\left(95 \% \mathrm{CI}=0.81-0.93, \mathrm{I}^{2}=0 \%\right)$, respectively (Figure 5). Median OS and PFS were 24.6-28.2 months and 18.2-18.3 months, respectively (Table 2).

\section{Monoclonal antibodies with unfavorable results}

Cixutumumab, figitumumab, carlumab, trastuzumab, LFA102, rilotumumab, and siltuximab did not show antitumor activity in early phase trials (Table 3) [12,14,45-50].

\begin{tabular}{|c|c|c|c|c|c|}
\hline Author & $\begin{array}{l}\text { Trial } \\
\text { phase }\end{array}$ & Drug combination & $\begin{array}{l}\text { Target of } \\
\text { MoAB }\end{array}$ & Problem & Outcomes \\
\hline $\begin{array}{l}\text { McHugh et al. } \\
(2020)[45]\end{array}$ & $\begin{array}{l}\text { Phase } \\
\text { I }\end{array}$ & $\begin{array}{l}\text { Cixutumumab + } \\
\text { temsirolimus }\end{array}$ & IGF-1R & $\begin{array}{l}\text { Metastatic } \\
\text { CRPC }\end{array}$ & $\begin{array}{l}\text { The combination therapy had limited anti-tumor } \\
\text { activity and a greater than expected toxicity }\end{array}$ \\
\hline $\begin{array}{l}\text { De Bono et al. } \\
\text { (2014) [46] }\end{array}$ & $\begin{array}{l}\text { Phase } \\
\text { II }\end{array}$ & $\begin{array}{l}\text { Figitumumab + } \\
\text { docetaxel }\end{array}$ & IGF-1R & $\begin{array}{l}\text { Metastatic } \\
\text { CRPC }\end{array}$ & $\begin{array}{l}\text { No significant PSA response. The combination not } \\
\text { recommended by authors in Bono et al. }\end{array}$ \\
\hline $\begin{array}{l}\text { Boudadi et al. } \\
\text { (2018) [48] }\end{array}$ & $\begin{array}{l}\text { Phase } \\
\text { II }\end{array}$ & Ipilimumab + nivolumab & $\begin{array}{l}\text { CTLA-4, } \\
\text { PD-1 }\end{array}$ & $\begin{array}{l}\text { Metastatic } \\
\text { CRPC }\end{array}$ & $\begin{array}{l}\text { Anti-tumor activity was only seen in patients with AR- } \\
\text { V7 isoform of the androgen receptor. Tumor activity } \\
\text { was not seen in other patients }\end{array}$ \\
\hline $\begin{array}{l}\text { Pienta et al. (2013) } \\
\text { [47] }\end{array}$ & $\begin{array}{l}\text { Phase } \\
\text { II }\end{array}$ & Carlumab & MCP-1 & $\begin{array}{l}\text { Metastatic } \\
\text { CRPC }\end{array}$ & $\begin{array}{l}\text { Well tolerated but did not show anti-tumor activity as a } \\
\text { single agent }\end{array}$ \\
\hline $\begin{array}{l}\text { Fizazi et al. (2012) } \\
\text { [12] }\end{array}$ & $\begin{array}{l}\text { Phase } \\
\text { II }\end{array}$ & $\begin{array}{l}\text { Siltuximab }+ \\
\text { mitoxantrone/prednisone }\end{array}$ & IL-6 & $\begin{array}{l}\text { Metastatic } \\
\text { CRPC }\end{array}$ & $\begin{array}{l}\text { The drug combination was well tolerated, } \\
\text { improvement in outcomes was not demonstrated }\end{array}$ \\
\hline $\begin{array}{l}\text { Ziada et al. (2004) } \\
\text { (NCT00003740) } \\
{[14]}\end{array}$ & $\begin{array}{l}\text { Phase } \\
\text { II }\end{array}$ & Trastuzumab & HER2 & CRPC & Well tolerated with no anti-tumor activity \\
\hline $\begin{array}{l}\text { Minami et al. } \\
(2020) \\
(\text { NCT01610050) } \\
\text { [49] }\end{array}$ & $\begin{array}{l}\text { Phase } \\
\text { I }\end{array}$ & LFA102 & $\begin{array}{l}\text { Anti- } \\
\text { prolactin } \\
\text { receptor }\end{array}$ & $\begin{array}{l}\text { Metastatic } \\
\text { CRPC }\end{array}$ & Well tolerated with no anti-tumor activity \\
\hline $\begin{array}{l}\text { Ryan et al. (2013) } \\
\text { (NCT00770848) } \\
{[50]}\end{array}$ & $\begin{array}{l}\text { Phase } \\
\text { I/II }\end{array}$ & AMG 102 (rilotumumab) & $\begin{array}{l}\text { Hepatocyte } \\
\text { growth } \\
\text { factor }\end{array}$ & $\begin{array}{l}\text { Resistant } \\
\text { CRPC }\end{array}$ & Well tolerated with no anti-tumor activity \\
\hline
\end{tabular}

\section{TABLE 3: Early phase trials on monoclonal antibodies with no anti-tumor activity.}

$\mathrm{MoAb}=$ monoclonal antibody; IGF-1R = insulin-like growth factor-1 receptor; CRPC = castration-resistant prostate cancer; PSA = prostate-specific antigen; CTLA-4 = cytotoxic T-lymphocyte-associated antigen-4; PD-1 = programmed cell death protein 1; MCP-1 = monocyte chemotactic protein-1; IL-6 = interleukin 6 ; HER2 = human epidermal growth factor receptor 2 .

\section{Ongoing clinical trials and interim results of ongoing trials}

Interim results of ongoing clinical trials on pembrolizumab, avelumab, atezolizumab, pasotuxizumab, and tremelimumab have shown promising results alone or in combination with chemotherapy [51-58]. Combinations are given in Table 4. 


\section{Cureus}

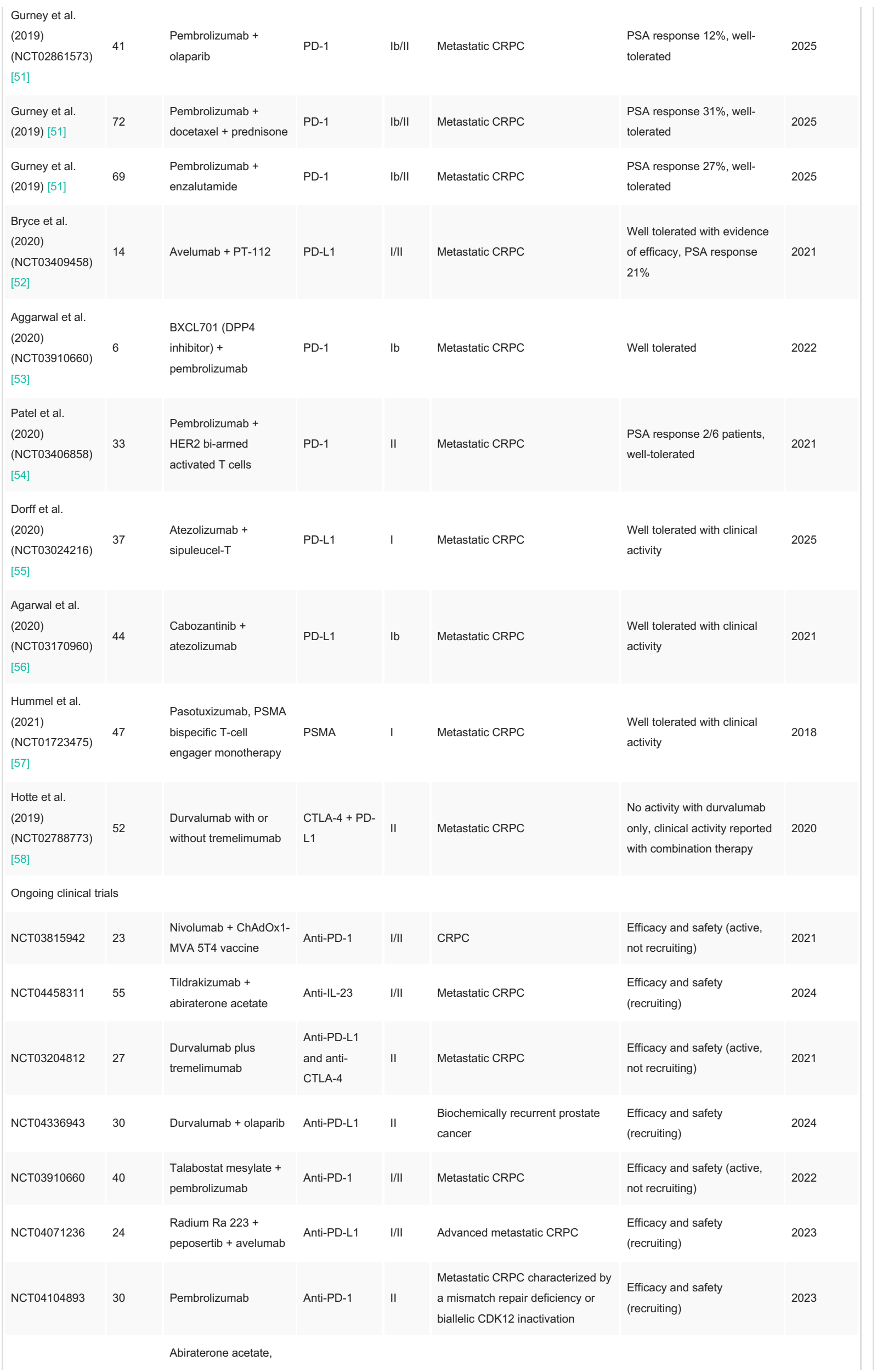




\section{Cureus}

\begin{tabular}{|c|c|c|c|c|c|c|c|}
\hline NCT02703623 & 198 & $\begin{array}{l}\text { apalutamide, } \\
\text { prednisone +/- } \\
\text { ipilimumab }\end{array}$ & Anti-CTLA-4 & II & Metastatic CRPC & $\begin{array}{l}\text { Efficacy and safety (active, } \\
\text { not recruiting) }\end{array}$ & 2022 \\
\hline NCT04159896 & 49 & ESK981 + nivolumab & Anti-PD-1 & II & Metastatic CRPC & $\begin{array}{l}\text { Efficacy and safety } \\
\text { (recruiting) }\end{array}$ & 2022 \\
\hline NCT03367819 & 134 & $\begin{array}{l}\text { Isatuximab + } \\
\text { cemiplimab }\end{array}$ & $\begin{array}{l}\text { Anti-CD-38 } \\
\text { and Anti-PD-1 }\end{array}$ & $\mathrm{I} / \mathrm{II}$ & Metastatic CRPC & $\begin{array}{l}\text { Efficacy and safety (active, } \\
\text { not recruiting) }\end{array}$ & 2021 \\
\hline NCT03805594 & 43 & $\begin{array}{l}\text { Lutetium Lu 177- } \\
\text { PSMA-617 + } \\
\text { pembrolizumab }\end{array}$ & $\begin{array}{l}\text { Anti-PSMA + } \\
\text { anti-PD-1 }\end{array}$ & 1 & Metastatic CRPC & $\begin{array}{l}\text { Efficacy and safety } \\
\text { (recruiting) }\end{array}$ & 2022 \\
\hline NCT02499835 & 66 & $\begin{array}{l}\text { Vaccine therapy }+ \\
\text { pembrolizumab }\end{array}$ & Anti-PD-1 & $\mathrm{I} / \mathrm{II}$ & Metastatic CRPC & $\begin{array}{l}\text { Efficacy and safety (active, } \\
\text { not recruiting) }\end{array}$ & 2021 \\
\hline NCT04471974 & 54 & $\begin{array}{l}\text { Pembrolizumab + ZEN- } \\
3694 \text { + enzalutamide }\end{array}$ & Anti-PD-1 & II & Metastatic CRPC & $\begin{array}{l}\text { Efficacy and safety } \\
\text { (recruiting) }\end{array}$ & 2025 \\
\hline NCT04592237 & 120 & $\begin{array}{l}\text { Cetrelimab + } \\
\text { cabazitaxel + } \\
\text { carboplatin + niraparib }\end{array}$ & Anti-PD-1 & II & Aggressive prostate cancer & $\begin{array}{l}\text { Efficacy and safety } \\
\text { (recruiting) }\end{array}$ & 2025 \\
\hline NCT02312557 & 58 & $\begin{array}{l}\text { Pembrolizumab + } \\
\text { enzalutamide }\end{array}$ & Anti-PD-1 & II & Metastatic CRPC & $\begin{array}{l}\text { Efficacy and safety (active, } \\
\text { not recruiting) }\end{array}$ & 2022 \\
\hline NCT03217747 & 184 & $\begin{array}{l}\text { PF-04518600 + } \\
\text { avelumab + } \\
\text { utomilumab }\end{array}$ & $\begin{array}{l}\text { Anti-OX40, } \\
\text { anti-PDL1, } \\
\text { and anti- } \\
\text { CD137 }\end{array}$ & $\mathrm{I} / \mathrm{II}$ & $\begin{array}{l}\text { Patients with advanced } \\
\text { malignancies }\end{array}$ & $\begin{array}{l}\text { Efficacy and safety } \\
\text { (recruiting) }\end{array}$ & 2023 \\
\hline NCT02601014 & 15 & Ipilimumab + nivolumab & $\begin{array}{l}\text { Anti-CTLA-4 } \\
\text { and anti-PD-1 }\end{array}$ & II & $\begin{array}{l}\text { AR-V7-expressing metastatic } \\
\text { CRPC }\end{array}$ & $\begin{array}{l}\text { Efficacy and safety (active, } \\
\text { not recruiting) }\end{array}$ & 2022 \\
\hline NCT04068896 & 90 & NGM120 & $\begin{array}{l}\text { GFRAL } \\
\text { antagonist } \\
\text { blocking } \\
\text { GDF15 }\end{array}$ & I & Metastatic CRPC & $\begin{array}{l}\text { Efficacy and safety } \\
\text { (recruiting) }\end{array}$ & 2021 \\
\hline NCT03849469 & 242 & $\begin{array}{l}\text { Pembrolizumab + } \\
\text { XmAb22841 }\end{array}$ & $\begin{array}{l}\text { Anti-PD-1 + } \\
\text { anti-CTLA-4 }\end{array}$ & I & $\begin{array}{l}\text { Selected advanced solid tumors } \\
\text { (DUET-4) }\end{array}$ & $\begin{array}{l}\text { Efficacy and safety } \\
\text { (recruiting) }\end{array}$ & 2027 \\
\hline NCT03517488 & 154 & XmAb20717 & $\begin{array}{l}\text { Anti-PD- } \\
\text { 1/anti-CTLA-4 }\end{array}$ & I & Advanced solid tumors & $\begin{array}{l}\text { Efficacy and safety } \\
\text { (recruiting) }\end{array}$ & 2021 \\
\hline NCT03454451 & 378 & $\begin{array}{l}\text { CPI-006 + } \\
\text { pembrolizumab }\end{array}$ & $\begin{array}{l}\text { Anti CD73 + } \\
\text { anti-PD-1 }\end{array}$ & I & Metastatic CRPC & $\begin{array}{l}\text { Efficacy and safety } \\
\text { (recruiting) }\end{array}$ & 2023 \\
\hline NCT03330405 & 216 & Avelumab + talazoparib & Anti-PD-L1 & II & CRPC & $\begin{array}{l}\text { Efficacy and safety (active, } \\
\text { not recruiting) }\end{array}$ & 2021 \\
\hline NCT04423029 & 260 & Nivolumab + DF6002 & Anti-PD-1 & $1 / I I$ & Metastatic solid tumors & $\begin{array}{l}\text { Efficacy and safety } \\
\text { (recruiting) }\end{array}$ & 2024 \\
\hline NCT03207867 & 376 & PDR001 + NIR178 & Anti-PD-1 & II & $\begin{array}{l}\text { CRPC, solid tumors, and } \\
\text { lymphoma }\end{array}$ & $\begin{array}{l}\text { Efficacy and safety } \\
\text { (recruiting) }\end{array}$ & 2021 \\
\hline NCT03983954 & 45 & $\begin{array}{l}\text { Naptumomab + } \\
\text { durvalumab }\end{array}$ & $\begin{array}{l}\text { Anti-5T4 and } \\
\text { anti-PD-L1 }\end{array}$ & I & $\begin{array}{l}\text { Solid tumor that is } \\
\text { metastatic/advanced }\end{array}$ & $\begin{array}{l}\text { Efficacy and safety } \\
\text { (recruiting) }\end{array}$ & 2022 \\
\hline NCT03970382 & 148 & Nivolumab & Anti-PD-1 & I & $\begin{array}{l}\text { Locally advanced or metastatic } \\
\text { solid tumors }\end{array}$ & $\begin{array}{l}\text { Efficacy and safety } \\
\text { (recruiting) }\end{array}$ & 2024 \\
\hline
\end{tabular}

\section{TABLE 4: Ongoing clinical trials and interim results of ongoing trials presented in conferences.}

$\mathrm{NCT}=$ National Clinical Trial; DPP4 = dipeptidyl peptidase 4; HER2 = human epidermal growth factor receptor 2; PD-1 = programmed cell death protein 1; PD-L1 = programmed death-ligand 1; CTLA-4 = cytotoxic T-lymphocyte-associated antigen-4; IL-23 = interleukin 23; CRPC = castration-resistant prostate cancer; PSA = prostate-specific antigen; $\mathrm{PSMA}=$ prostate-specific membrane antigen. 


\section{Discussion}

Docetaxel is the most used chemotherapy-based treatment for metastatic CRPC as docetaxel improved OS, PFS, and PSA levels in RCT [59]. Among non-chemotherapy drugs, alpharadin, abiraterone, radium-223 dichloride, etc., showed improvement in survival rates with anti-tumor activity [60]. Among immunotherapies, sipuleucel-T extended OS without improving PFS [61]. However, these therapies are not curative, responses are rarely durable, and are poorly tolerated by some patients. Additional treatment options are needed for better outcomes. In RCTs, majorly monoclonal antibodies were used in combination with docetaxel or in patients refractory to docetaxel therapy. According to the pooled results, monoclonal antibodies improved PFS and PSA response as compared to placebo.

Checkpoint inhibitors, including PD-1, programmed death-ligand 1 (PD-L1), and CTLA-4 inhibitors, have shown efficacy in urothelial and other solid tumors [62-65]. However, the microenvironment of prostate cancer is more immunosuppressive as compared to other tumors [66,67]. Ipilimumab (CTLA-4 inhibitor) improved PFS and PSA levels in both trials, including docetaxel pre-treated and treatment naïve patients. It was well tolerated in both trials. OS was not prolonged on normal follow-up. However, long-term follow-up of five years showed better OS in the ipilimumab group as compared to placebo [68]. More trials are now conducted on combination therapy of ipilimumab. In the trial conducted by Boudadi et al. (2018) [48], 1 $\mathrm{mg} / \mathrm{kg}$ of ipilimumab was used with nivolumab and anti-tumor activity was only reported in a small group of patients. However, according to the preliminary results of a trial by Sharma et al. (2020), $3 \mathrm{mg}$ of ipilimumab with nivolumab showed anti-tumor activity in all subsets of patients and a large-scale phase II trial is in progress on ipilimumab + nivolumab in metastatic CRPC patients [16]. Another RCT is in progress to assess the efficacy and safety of ipilimumab in combination with abiraterone acetate, apalutamide, and prednisone. Ongoing clinical trials are also testing nivolumab in combination with ChAdOx1-MVA 5T4 vaccine, ESK981 (Pan-VEGFR/Tie2 tyrosine kinase inhibitor), and DF6002 (binds interleukin 12 (IL-12) receptor).

In a multicohort phase II trial by Antonarakis et al. (2020), pembrolizumab showed anti-tumor activity in docetaxel pretreated patients and the observed survival estimates are promising [17]. Although $5 \%$ of the patients showed OR, the response was durable. Pembrolizumab monotherapy was well tolerated, and no unexpected toxicities were reported. A combination of pembrolizumab with olaparib, enzalutamide, and docetaxel is tested in KEYNOTE-365, and the early results have shown anti-tumor activity of these combinations and are well tolerated [51]. According to the results of a phase II trial by Graff et al. (2020), pembrolizumab addition to enzalutamide showed anti-tumor activity in patients refractory to enzalutamide alone, and the response was durable [42]. Another trial was conducted on the addition of pembrolizumab to the anti-tumor DNA vaccine. The addition of pembrolizumab showed better results in terms of PSA decline, $\mathrm{OR}$, and $\mathrm{CD}-8+\mathrm{T}$ cell infiltration into tumor lesions as compared to vaccination alone. More trials are in progress to assess the efficacy and safety of pembrolizumab in combination with dipeptidyl peptidase 4 (DPP4) inhibitor BXCL701, HER2 bi-armed activated T cells, talabostat mesylate, lutetium lu 177-PSMA-617, vaccine therapy, ZEN-3694 + enzalutamide, enzalutamide, XmAb22841, and CPI-006 (Table 4). Avelumab, atezolizumab, tremelimumab, cemiplimab, cetrelimab, XmAb20717, PDR001, and durvalumab are other checkpoint inhibitors that are getting tested alone and in combination therapy for the treatment of CRPC.

The anti-angiogenic drug, bevacizumab, also improved PFS and PSA levels without any improvement in OS. Bevacizumab was also tested in combination regimens. Among the combination regimens, the four-drug regimen of bevacizumab with docetaxel + thalidomide + prednisone and lenalidomide + docetaxel + prednisone showed the best efficacy outcomes, and toxicities were manageable (Figure 5 and Table 2). Early anti-tumor activity was reported with the addition of thalidomide and bevacizumab to docetaxel as compared to docetaxel alone. Bevacizumab in combination with satraplatin has shown promising results in early phase trials in docetaxel refractory patients. The addition of everolimus (mammalian target of rapamycin (mTOR) inhibitor) to docetaxel + bevacizumab did not show better outcomes as compared to docetaxel + bevacizumab in the early-phase trial.

Abituzumab improved the progression of the disease, but the results were not statistically significant. In our analysis, the trial with intetumumab was the outlier and intetumumab caused worsening in PFS or PSA levels. However, intetumumab did not increase adverse events as compared to placebo. Intetumumab might have interacted with docetaxel, resulting in lower efficacy.

Lack of improvement in OS despite changes in PFS and PSA levels might be due to the unique response of CRPC to these drugs. Also, the patients with metastatic CRPC are generally older than patients with other types of cancer, e.g., breast cancer and lung cancer, and comparatively more patients have bone metastasis [69-71]. Other possible explanations can be the unique mechanism of action of these drugs or flaws in trial designs. These drugs might show some improvement in OS if followed for longer durations. Further studies should be conducted on how to utilize the anti-tumor activity of these monoclonal antibodies.

Denosumab targets receptor activator of nuclear factor kappa-B ligand (RANKL) and is an anti-bone resorptive agent. It delayed skeletal-related adverse events as compared to zoledronic acid in patients with CRPC with bone metastasis in RCT. Zoledronic acid was proved better than a placebo in an RCT [72]. However, increased incidence of osteonecrosis of the jaw was associated with denosumab as compared to 


\section{Cureus}

zoledronic acid. A meta-analysis showed similar results for denosumab in the prevention of skeletal-related adverse events as compared to zoledronic acid [73]. Moreover, an RCT by Smith et al. (2012) tested denosumab for the prevention of bone metastasis [25]. Denosumab significantly improved bone metastasisfree survival and time to first bone metastasis as compared to placebo. The major adverse event observed in the denosumab group was the osteonecrosis of the jawbone.

In a non-comparative randomized study, cixutumumab (IGF-1R inhibitor) and ramucirumab (VEGFR inhibitor) were used with mitoxantrone-prednisone. PFS in the cixutumumab group was similar to the projected value, while ramucirumab showed better PFS as compared to the projected value (6.7 months vs. 3.9 months). The incidence of adverse events was similar to expectations. Ramucirumab has shown improvement in OS in RCTs on other solid tumors [74]. Another trial by McHugh et al. (2020) has also shown no activity of cixutumumab with temsirolimus [45].

Among monoclonal antibodies, PD-1 inhibitors, PD-L1 inhibitors, and CTLA-4 inhibitors have the potential to become the drugs of the future for patients with prostate cancer. More multicenter randomized clinical trials should focus on finding the efficacy and appropriate combination of these medications. However, the role of monoclonal antibodies in prostate cancer is still debated.

\section{Conclusions}

Monoclonal antibodies were well tolerated and showed better outcomes in terms of PFS and $>50 \%$ decline in PSA levels compared to placebo. However, OS was only improved with ipilimumab as compared to placebo on long-term follow-up of five years. Denosumab delayed skeletal-related adverse events as compared to zoledronic acid in CRPC with bone metastasis. Denosumab also delayed bone metastasis as compared to placebo in patients with metastatic CRPC. Pembrolizumab, avelumab, atezolizumab, pasotuxizumab, and tremelimumab have shown promising results in the early phase trials. More multicenter, double-blind clinical trials may be needed to confirm these results.

\section{Appendices}




\section{Cureus}

$\mathbf{P}$

"Prostatic Neoplasms, Castration-Resistant"[Mesh]

"Antibodies,

Monoclonal" [Mesh]

Prostatic Neoplasms, Castration-Resistant Castration-Resistant Prostatic Neoplasm Prostatic Neoplasms, Castration-Resistant Androgen-Insensitive Prostatic Neoplasms Androgen Insensitive Prostatic Neoplasms Androgen-Resistant Prostatic Neoplasms Androgen Resistant Prostatic Neoplasms Prostatic Neoplasms, Hormone Refractory Hormone Refractory Prostatic Neoplasms Prostatic Neoplasms, Androgen-Independent Neoplasm, Androgen-Independent Prostatic Prostatic Neoplasm, Androgen-Independent Prostatic Neoplasms, Androgen Independent Prostatic Neoplasms, Androgen-Insensitive Androgen-Insensitive Prostatic Neoplasm Prostatic Neoplasms, Androgen Insensitive Prostatic Neoplasms, Androgen-Resistant Androgen-Resistant Prostatic Neoplasm Prostatic Neoplasm, Androgen-Resistant Prostatic Neoplasms, Androgen Resistant Androgen-Independent Prostatic Neoplasms Androgen Independent Prostatic Neoplasms Castration-Resistant Prostatic Neoplasms Castration-Resistant Prostatic Neoplasms Cancers, Castration-Resistant Prostatic Androgen-Insensitive Prostatic Cancer Androgen Insensitive Prostatic Cancer Androgen-Resistant Prostatic Cancer Androgen Resistant Prostatic Cancer Prostatic Cancer, Hormone Refractory Prostatic Cancer, Androgen-Independent Androgen-Independent Prostatic Cancers Prostatic Cancer, Androgen Independent Prostatic Cancers, Androgen-Independent Prostatic Cancer, Androgen-Insensitive Androgen-Insensitive Prostatic Cancers Cancer, Androgen-Insensitive Prostatic Cancers, Androgen-Insensitive Prostatic Prostatic Cancer, Androgen Insensitive Prostatic Cancers, Androgen-Insensitive Prostatic Cancer, Androgen-Resistant Androgen-Resistant Prostatic Cancers Cancer, Androgen-Resistant Prostatic Cancers, Androgen-Resistant Prostatic Prostatic Cancer, Androgen Resistant

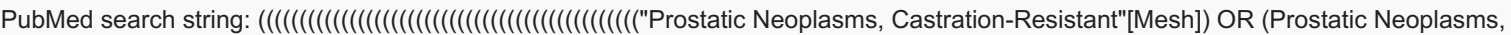
Castration-Resistant)) OR (Castration-Resistant Prostatic Neoplasm)) OR (Prostatic Neoplasms, Castration Resistant)) OR (AndrogenInsensitive Prostatic Neoplasms)) OR (Androgen Insensitive Prostatic Neoplasms)) OR (Androgen-Resistant Prostatic Neoplasms)) OR (Androgen Resistant Prostatic Neoplasms)) OR (Prostatic Neoplasms, Hormone Refractory)) OR (Hormone Refractory Prostatic Neoplasms)) OR (Prostatic Neoplasms, Androgen-Independent)) OR (Neoplasm, Androgen-Independent Prostatic)) OR (Prostatic Neoplasm, Androgen-Independent)) OR (Prostatic Neoplasms, Androgen Independent)) OR (Prostatic Neoplasms, Androgen-Insensitive)) OR (Androgen-Insensitive Prostatic Neoplasm)) OR (Prostatic Neoplasms, Androgen Insensitive)) OR (Prostatic Neoplasms, AndrogenResistant)) OR (Androgen-Resistant Prostatic Neoplasm)) OR (Prostatic Neoplasm, Androgen-Resistant)) OR (Prostatic Neoplasms, Androgen Resistant)) OR (Androgen-Independent Prostatic Neoplasms)) OR (Androgen Independent Prostatic Neoplasms)) OR (Castration-Resistant Prostatic Neoplasms)) OR (Castration Resistant Prostatic Neoplasms)) OR (Cancers, Castration-Resistant Prostatic)) OR (Androgen-Insensitive Prostatic Cancer)) OR (Androgen Insensitive Prostatic Cancer)) OR (Androgen-Resistant Prostatic Cancer)) OR (Androgen Resistant Prostatic Cancer)) OR (Prostatic Cancer, Hormone Refractory)) OR (Prostatic Cancer, AndrogenIndependent)) OR (Androgen-Independent Prostatic Cancers)) OR (Prostatic Cancer, Androgen Independent)) OR (Prostatic Cancers, Androgen-Independent)) OR (Prostatic Cancer, Androgen-Insensitive)) OR (Androgen-Insensitive Prostatic Cancers)) OR (Cancer, Androgen-Insensitive Prostatic)) OR (Cancers, Androgen-Insensitive Prostatic)) OR (Prostatic Cancer, Androgen Insensitive)) OR (Prostatic Cancers, Androgen-Insensitive)) OR (Prostatic Cancer, Androgen-Resistant)) OR (Androgen-Resistant Prostatic Cancers)) OR (Cancer, Androgen-Resistant Prostatic)) OR (Cancers, Androgen-Resistant Prostatic)) OR (Prostatic Cancer, Androgen Resistant)) AND ((((("Antibodies, Monoclonal"[Mesh]) OR (Monoclonal Antibodies)) OR (Monoclonal Antibody)) OR (Antibody, Monoclonal)) AND $((((()((()($ "Prostatic Neoplasms"[Mesh]) OR (Prostatic Neoplasms)) OR (Neoplasms, Prostate)) OR (Prostate Neoplasm)) OR (Neoplasms, Prostatic)) OR (Prostatic Neoplasm)) OR (Prostate Cancer)) OR (Prostate Cancers)) OR (Cancer of the Prostate)) OR (Prostatic Cancer)) OR (Prostatic Cancers)) OR (Cancer of Prostate))) $=424$

Embase search string: ('castration resistant prostate cancer'/exp OR 'crpc (castration resistant prostate cancer)' OR 'castrate resistant prostate cancer' OR 'castration resistant prostate cancer' OR 'castration-resistant pc' OR 'castration-resistant pca' OR 'castration-resistant prostatic neoplasms' OR 'hormone refractory prostate cancer' OR 'prostatic neoplasms, castration-resistant') AND ('monoclonal antibody'/exp OR 'antibodies, monoclonal' OR 'antibodies, monoclonal, humanized' OR 'antibodies, monoclonal, murine derived' OR 'antibodies, monoclonal, murine-derived' OR 'antibody, monoclonal' OR 'clonal antibody' OR 'hybridoma antibody' OR 'monoclonal antibodies' OR 'monoclonal antibody') = 2,427

Web of Science: with keywords mentioned above $=49$

Cochrane: with keywords mentioned above $=60$

ClinicalTrials.gov: prostate cancer + monoclonal antibodies $=109$

Total $=2,960$

TABLE 5: Keywords and search strings. 


\section{Additional Information \\ Disclosures}

Human subjects: All authors have confirmed that this study did not involve human participants or tissue. Animal subjects: All authors have confirmed that this study did not involve animal subjects or tissue. Conflicts of interest: In compliance with the ICMJE uniform disclosure form, all authors declare the following: Payment/services info: All authors have declared that no financial support was received from any organization for the submitted work. Financial relationships: All authors have declared that they have no financial relationships at present or within the previous three years with any organizations that might have an interest in the submitted work. Other relationships: All authors have declared that there are no other relationships or activities that could appear to have influenced the submitted work.

\section{References}

1. Siegel RL, Miller KD, Jemal A: Cancer statistics, 2020. CA Cancer J Clin. 2020, 70:7-30. 10.3322/caac.21590

2. Siegel RL, Miller KD, Jemal A: Cancer statistics, 2019. CA Cancer J Clin. 2019, 69:7-34. 10.3322/caac.21551

3. Rawla P: Epidemiology of prostate cancer. World J Oncol. 2019, 10:63-89. 10.14740/wjon1191

4. Barsouk A, Padala SA, Vakiti A, et al.: Epidemiology, staging and management of prostate cancer . Med Sci (Basel). 2020, 8:28. 10.3390/medsci8030028

5. Mottet N, Bellmunt J, Bolla M, et al.: EAU-ESTRO-SIOG guidelines on prostate cancer. Part 1: screening, diagnosis, and local treatment with curative intent. Eur Urol. 2017, 71:618-29. 10.1016/j.eururo.2016.08.003

6. Heidenreich A, Bastian PJ, Bellmunt J, et al.: EAU guidelines on prostate cancer. Part II: treatment of advanced, relapsing, and castration-resistant prostate cancer. Eur Urol. 2014, 65:467-79. 10.1016/j.eururo.2013.11.002

7. Cookson MS, Roth BJ, Dahm P, et al.: Castration-resistant prostate cancer: AUA guideline . J Urol. 2013, 190:429-38. 10.1016/j.juro.2013.05.005

8. Kirby M, Hirst C, Crawford ED: Characterising the castration-resistant prostate cancer population: a systematic review. Int J Clin Pract. 2011, 65:1180-92. 10.1111/j.1742-1241.2011.02799.x

9. Cassinello J, Arranz JÁ, Piulats JM, et al.: SEOM clinical guidelines for the treatment of metastatic prostate cancer (2017). Clin Transl Oncol. 2018, 20:57-68. 10.1007/s12094-017-1783-2

10. Kazazi-Hyseni F, Beijnen JH, Schellens JH: Bevacizumab. Oncologist. 2010, 15:819-25. 10.1634/theoncologist.2009-0317

11. Gross ME, Dorff TB, Quinn DI, Diaz PM, Castellanos OO, Agus DB: Safety and efficacy of docetaxel, bevacizumab, and everolimus for castration-resistant prostate cancer (CRPC). Clin Genitourin Cancer. 2017, 16:e11-21. 10.1016/j.clgc.2017.07.003

12. Fizazi K, De Bono JS, Flechon A, et al.: Randomised phase II study of siltuximab (CNTO 328), an anti-IL-6 monoclonal antibody, in combination with mitoxantrone/prednisone versus mitoxantrone/prednisone alone in metastatic castration-resistant prostate cancer. Eur J Cancer. 2012, 48:85-93. 10.1016/j.ejca.2011.10.014

13. Hussain M, Le Moulec S, Gimmi C, Bruns R, Straub J, Miller K: Differential effect on bone lesions of targeting integrins: randomized phase II trial of abituzumab in patients with metastatic castration-resistant prostate cancer. Clin Cancer Res. 2016, 22:3192-200. 10.1158/1078-0432.CCR-15-2512

14. Ziada A, Barqawi A, Glode LM, et al.: The use of trastuzumab in the treatment of hormone refractory prostate cancer; phase II trial. Prostate. 2004, 60:332-7. 10.1002/pros.20065

15. Cathomas R, Rothermundt C, Klingbiel D, et al.: Efficacy of cetuximab in metastatic castration-resistant prostate cancer might depend on EGFR and PTEN expression: results from a phase II trial (SAKK 08/07). Clin Cancer Res. 2012, 18:6049-57. 10.1158/1078-0432.CCR-12-2219

16. Sharma P, Pachynski RK, Narayan V, et al.: Nivolumab plus ipilimumab for metastatic castration-resistant prostate cancer: preliminary analysis of patients in the CheckMate 650 trial. Cancer Cell. 2020, 38:48999.e3. 10.1016/j.ccell.2020.08.007

17. Antonarakis ES, Piulats JM, Gross-Goupil M, et al.: Pembrolizumab for treatment-refractory metastatic castration-resistant prostate cancer: multicohort, open-label phase II KEYNOTE-199 study. J Clin Oncol. 2020, 38:395-405. 10.1200/JCO.19.01638

18. Hegemann M, Bedke J, Stenzl A, Todenhöfer T: Denosumab treatment in the management of patients with advanced prostate cancer: clinical evidence and experience. Ther Adv Urol. 2017, 9:81-8. $10.1177 / 1756287216686018$

19. Higgins JPT, Thomas J, Chandler J, Cumpston M, Li T, Page MJ, Welch VA: Cochrane Handbook for Systematic Reviews of Interventions. Wiley, Hoboken, NJ; 2019. 10.1002/9781119536604

20. Liberati A, Altman DG, Tetzlaff J, et al.: The PRISMA statement for reporting systematic reviews and metaanalyses of studies that evaluate healthcare interventions: explanation and elaboration. BMJ. 2009, 339:b2700. 10.1136/bmi.b2700

21. Schardt C, Adams MB, Owens T, Keitz S, Fontelo P: Utilization of the PICO framework to improve searching PubMed for clinical questions. BMC Med Inform Decis Mak. 2007, 7:16. 10.1186/1472-6947-7-16

22. Sterne JA, Savović J, Page MJ, et al.: RoB 2: a revised tool for assessing risk of bias in randomised trials . BMJ. 2019, 366:14898. 10.1136/bmj.14898

23. Balduzzi S, Rücker G, Schwarzer G: How to perform a meta-analysis with R: a practical tutorial . Evid Based Ment Health. 2019, 22:153-60. 10.1136/ebmental-2019-300117

24. Beer TM, Kwon ED, Drake CG, et al.: Randomized, double-blind, phase III trial of ipilimumab versus placebo in asymptomatic or minimally symptomatic patients with metastatic chemotherapy-naive castrationresistant prostate cancer. J Clin Oncol. 2017, 35:40-7. 10.1200/JCO.2016.69.1584

25. Smith MR, Saad F, Coleman R, et al.: Denosumab and bone-metastasis-free survival in men with castrationresistant prostate cancer: results of a phase 3, randomised, placebo-controlled trial. Lancet. 2012, 379:3946. 10.1016/S0140-6736(11)61226-9

26. Fizazi K, Carducci M, Smith M, et al.: Denosumab versus zoledronic acid for treatment of bone metastases in 
men with castration-resistant prostate cancer: a randomised, double-blind study. Lancet. 2011, 377:813-22. 10.1016/S0140-6736(10)62344-6

27. Heidenreich A, Rawal SK, Szkarlat K, et al.: A randomized, double-blind, multicenter, phase 2 study of a human monoclonal antibody to human $\alpha \nu$ integrins (intetumumab) in combination with docetaxel and prednisone for the first-line treatment of patients with metastatic castration-resistant prostate cancer. Ann Oncol. 2013, 24:329-36. 10.1093/annonc/mds505

28. Kwon ED, Drake CG, Scher HI, et al.: Ipilimumab versus placebo after radiotherapy in patients with metastatic castration-resistant prostate cancer that had progressed after docetaxel chemotherapy (CA184043): a multicentre, randomised, double-blind, phase 3 trial. Lancet Oncol. 2014, 15:700-12. 10.1016/S14702045(14)70189-5

29. Kelly WK, Halabi S, Carducci M, et al.: Randomized, double-blind, placebo-controlled phase III trial comparing docetaxel and prednisone with or without bevacizumab in men with metastatic castrationresistant prostate cancer: CALGB 90401. J Clin Oncol. 2012, 30:1534-40. 10.1200/JCO.2011.39.4767

30. Hussain M, Rathkopf D, Liu G, et al.: A randomised non-comparative phase II trial of cixutumumab (IMCA12) or ramucirumab (IMC-1121B) plus mitoxantrone and prednisone in men with metastatic docetaxelpretreated castration-resistant prostate cancer. Eur J Cancer. 2015, 51:1714-24. 10.1016/j.ejca.2015.05.019

31. Vaishampayan U, Thakur A, Rathore R, Kouttab N, Lum LG: Phase I study of anti-CD3 x anti-Her2 bispecific antibody in metastatic castrate resistant prostate cancer patients. Prostate Cancer. 2015, 2015:285193. $10.1155 / 2015 / 285193$

32. Vaishampayan UN, Fontana J, Heilbrun LK, Smith D, Heath E, Dickow B, Figg WD: Phase II trial of bevacizumab and satraplatin in docetaxel-pretreated metastatic castrate-resistant prostate cancer. Urol Oncol. 2014, 32:31.e25-33. 10.1016/j.urolonc.2012.11.017

33. Picus J, Halabi S, Kelly WK, et al.: A phase 2 study of estramustine, docetaxel, and bevacizumab in men with castrate-resistant prostate cancer: results from Cancer and Leukemia Group B Study 90006. Cancer. 2011, 117:526-33. 10.1002/cncr.25421

34. McNeel DG, Eickhoff JC, Wargowski E, Zahm C, Staab MJ, Straus J, Liu G: Concurrent, but not sequential, PD-1 blockade with a DNA vaccine elicits anti-tumor responses in patients with metastatic, castrationresistant prostate cancer. Oncotarget. 2018, 9:25586-96. 10.18632/oncotarget.25387

35. Batra JS, Niaz MJ, Whang YE, et al.: Phase I trial of docetaxel plus lutetium-177-labeled anti-prostatespecific membrane antigen monoclonal antibody J591 (177Lu-J591) for metastatic castration-resistant prostate cancer. Urol Oncol. 2020, 38:848.e9-16. 10.1016/j.urolonc.2020.05.028

36. Madan RA, Karzai FH, Ning YM, et al.: Phase II trial of docetaxel, bevacizumab, lenalidomide and prednisone in patients with metastatic castration-resistant prostate cancer. BJU Int. 2016, 118:590-7. 10.1111/bju.13412

37. Slovin SF, Higano CS, Hamid O, et al.: Ipilimumab alone or in combination with radiotherapy in metastatic castration-resistant prostate cancer: results from an open-label, multicenter phase I/II study. Ann Oncol. 2013, 24:1813-21. 10.1093/annonc/mdt107

38. Barata PC, Cooney M, Mendiratta P, Gupta R, Dreicer R, Garcia JA: Phase I/II study evaluating the safety and clinical efficacy of temsirolimus and bevacizumab in patients with chemotherapy refractory metastatic castration-resistant prostate cancer. Invest New Drugs. 2019, 37:331-7. 10.1007/s10637-018-0687-5

39. Autio KA, Klebanoff CA, Schaer D, et al.: Immunomodulatory activity of a colony-stimulating factor-1 receptor inhibitor in patients with advanced refractory breast or prostate cancer: a phase I study. Clin Cancer Res. 2020, 26:5609-20. 10.1158/1078-0432.CCR-20-0855

40. Di Lorenzo G, Figg WD, Fossa SD, et al.: Combination of bevacizumab and docetaxel in docetaxel-pretreated hormone-refractory prostate cancer: a phase 2 study. Eur Urol. 2008, 54:1089-94. 10.1016/j.eururo.2008.01.082

41. Hudes G, Tagawa ST, Whang YE, et al.: A phase 1 study of a chimeric monoclonal antibody against interleukin-6, siltuximab, combined with docetaxel in patients with metastatic castration-resistant prostate cancer. Invest New Drugs. 2013, 31:669-76. 10.1007/s10637-012-9857-z

42. Graff JN, Antonarakis ES, Hoimes CJ, et al.: Pembrolizumab (pembro) plus enzalutamide (enza) for enzaresistant metastatic castration-resistant prostate cancer (mCRPC): KEYNOTE-199 cohorts 4-5. J Clin Oncol. 2020, 38:15. 10.1200/JCO.2020.38.6_suppl.15

43. Francini F, Pascucci A, Francini E, et al.: Bevacizumab and weekly docetaxel in patients with metastatic castrate-resistant prostate cancer previously exposed to docetaxel. Prostate Cancer. 2011, 2011:258689. $10.1155 / 2011 / 258689$

44. Ning YM, Gulley JL, Arlen PM, et al.: Phase II trial of bevacizumab, thalidomide, docetaxel, and prednisone in patients with metastatic castration-resistant prostate cancer. J Clin Oncol. 2010, 28:2070-6. 10.1200/TCO.2009.25.4524

45. McHugh DJ, Chudow J, DeNunzio M, et al.: A phase I trial of IGF-1R inhibitor cixutumumab and mTOR inhibitor temsirolimus in metastatic castration-resistant prostate cancer. Clin Genitourin Cancer. 2020, 18:171-8.e2. 10.1016/j.clgc.2019.10.013

46. de Bono JS, Piulats JM, Pandha HS, et al.: Phase II randomized study of figitumumab plus docetaxel and docetaxel alone with crossover for metastatic castration-resistant prostate cancer. Clin Cancer Res. 2014, 20:1925-34. 10.1158/1078-0432.CCR-13-1869

47. Pienta KJ, Machiels JP, Schrijvers D, et al.: Phase 2 study of carlumab (CNTO 888), a human monoclonal antibody against CC-chemokine ligand 2 (CCL2), in metastatic castration-resistant prostate cancer. Invest New Drugs. 2013, 31:760-8. 10.1007/s10637-012-9869-8

48. Boudadi K, Suzman DL, Anagnostou V, et al.: Ipilimumab plus nivolumab and DNA-repair defects in AR-V7expressing metastatic prostate cancer. Oncotarget. 2018, 9:28561-71. 10.18632/oncotarget.25564

49. Minami H, Ando Y, Tamura K, Tajima T, Isaacs R: Phase I study of LFA102 in patients with advanced breast cancer or castration-resistant prostate cancer. Anticancer Res. 2020, 40:5229-35. 10.21873/anticanres.14526

50. Ryan CJ, Rosenthal M, Ng S, et al.: Targeted MET inhibition in castration-resistant prostate cancer: a randomized phase II study and biomarker analysis with rilotumumab plus mitoxantrone and prednisone. Clin Cancer Res. 2013, 19:215-24. 10.1158/1078-0432.CCR-12-2605 
51. Gurney H, Massard C, Retz M, et al.: Pembrolizumab combination therapies in patients with metastatic castrate-resistant prostate cancer (mCRPC): cohorts A-C of the phase 1B/2 KEYNOTE-365 study. AsiaPacific Journal of Clinical Oncology. 2019, 15:31-2.

52. Bryce AH, Dronca RS, Costello BA, Infante JR, Ames TD, Jimeno J, Karp DD: PT-112 in advanced metastatic castrate-resistant prostate cancer (MCRPC), as monotherapy or in combination with PD-L1 inhibitor avelumab: findings from two phase I studies. J Clin Oncol. 2020, 38:83. 10.1200/JCO.2020.38.6_suppl.83

53. Aggarwal RR, Costin D, O'Neill VJ, et al.: Phase 1b study of BXCL701, a novel small molecule inhibitor of dipeptidyl peptidases (DPP), combined with pembrolizumab (pembro), in men with metastatic castrationresistant prostate cancer (mCRPC). J Clin Oncol. 2020, 38:e17581. 10.1200/JCO.2020.38.15_suppl.e17581

54. Patel M, Lum LG, Deol A, et al.: Phase II trial of a novel immunotherapy combination of pembrolizumab and HER2 bi-armed activated T cells (BATs) in metastatic castrate resistant prostate cancer. J Clin Oncol. 2020, 38:97. 10.1200/JCO.2020.38.6_suppl.97

55. Dorff TB, Acoba JD, Pal SK, Scholz MC, Tamura DJ, Huang J, Rosser CJ: Assessing different sequencing regimens of atezolizumab (atezo) and sipuleucel-T (sipT) in patients who have asymptomatic or minimally symptomatic metastatic castrate-resistant prostate cancer. J Clin Oncol. 2020, 38:141. 10.1200/JCO.2020.38.6_suppl.141

56. Agarwal N, Loriot Y, McGregor BA, et al.: Cabozantinib (C) in combination with atezolizumab (A) in patients (pts) with metastatic castration-resistant prostate cancer (mCRPC): results of cohort 6 of the COSMIC-021 study. J Clin Oncol. 2020, 38:139. 10.1200/JCO.2020.38.6_suppl.139

57. Hummel HD, Kufer P, Grüllich C, et al.: Pasotuxizumab, a BiTE ${ }^{\circledR}$ immune therapy for castration-resistant prostate cancer: phase I, dose-escalation study findings. Immunotherapy. 2021, 13:125-41. 10.2217/imt2020-0256

58. Hotte SJ, Winquist E, Chi KN, et al.: CCTG IND 232: a phase II study of durvalumab with or without tremelimumab in patients with metastatic castration resistant prostate cancer (mCRPC). Ann Oncol. 2019, 30:885. 10.1093/annonc/mdz394.044

59. Tannock IF, de Wit R, Berry WR, et al.: Docetaxel plus prednisone or mitoxantrone plus prednisone for advanced prostate cancer. N Engl J Med. 2004, 351:1502-12. 10.1056/NEJMoa040720

60. Hoskin P, Sartor O, O'Sullivan JM, et al.: Efficacy and safety of radium-223 dichloride in patients with castration-resistant prostate cancer and symptomatic bone metastases, with or without previous docetaxel use: a prespecified subgroup analysis from the randomised, double-blind, phase 3 ALSYMPCA trial. Lancet Oncol. 2014, 15:1397-406. 10.1016/S1470-2045(14)70474-7

61. Kantoff PW, Higano CS, Shore ND, et al.: Sipuleucel-T immunotherapy for castration-resistant prostate cancer. N Engl J Med. 2010, 363:411-22. 10.1056/NEJMoa1001294

62. Ali MA, Aiman W, Shah SS, Hussain M, Kashyap R: Efficacy and safety of pembrolizumab based therapies in triple-negative breast cancer: a systematic review of clinical trials. Crit Rev Oncol Hematol. 2021, 157:103197. 10.1016/j.critrevonc.2020.103197

63. Dafni U, Tsourti Z, Vervita K, Peters S: Immune checkpoint inhibitors, alone or in combination with chemotherapy, as first-line treatment for advanced non-small cell lung cancer. A systematic review and network meta-analysis. Lung Cancer. 2019, 134:127-40. 10.1016/j.lungcan.2019.05.029

64. Bellmunt J, de Wit R, Vaughn DJ, et al.: Pembrolizumab as second-line therapy for advanced urothelial carcinoma. N Engl J Med. 2017, 376:1015-26. 10.1056/NEJMoa1613683

65. Sharma P, Retz M, Siefker-Radtke A, et al.: Nivolumab in metastatic urothelial carcinoma after platinum therapy (CheckMate 275): a multicentre, single-arm, phase 2 trial. Lancet Oncol. 2017, 18:312-22. 10.1016/s1470-2045(17)30065-7

66. Pasero C, Gravis G, Guerin M, et al.: Inherent and tumor-driven immune tolerance in the prostate microenvironment impairs natural killer cell antitumor activity. Cancer Res. 2016, 76:2153-65. 10.1158/0008-5472.CAN-15-1965

67. Sfanos KS, Bruno TC, Maris CH, et al.: Phenotypic analysis of prostate-infiltrating lymphocytes reveals TH17 and Treg skewing. Clin Cancer Res. 2008, 14:3254-61. 10.1158/1078-0432.CCR-07-5164

68. Fizazi K, Drake CG, Beer TM, et al.: Final analysis of the ipilimumab versus placebo following radiotherapy phase III trial in postdocetaxel metastatic castration-resistant prostate cancer identifies an excess of longterm survivors. Eur Urol. 2020, 78:822-30. 10.1016/j.eururo.2020.07.032

69. Hellmann MD, Paz-Ares L, Bernabe Caro R, et al.: Nivolumab plus ipilimumab in advanced non-small-cell lung cancer. N Engl J Med. 2019, 381:2020-31. 10.1056/NEJMoa1910231

70. Sandler A, Gray R, Perry MC, et al.: Paclitaxel-carboplatin alone or with bevacizumab for non-small-cell lung cancer. N Engl J Med. 2006, 355:2542-50. 10.1056/NEJMoa061884

71. Miller K, Wang M, Gralow J, et al.: Paclitaxel plus bevacizumab versus paclitaxel alone for metastatic breast cancer. N Engl J Med. 2007, 357:2666-76. 10.1056/NEJMoa072113

72. Saad F, Gleason DM, Murray R, et al.: Long-term efficacy of zoledronic acid for the prevention of skeletal complications in patients with metastatic hormone-refractory prostate cancer. J Natl Cancer Inst. 2004, 96:879-82. 10.1093/jnci/djh141

73. Chen C, Li R, Yang T, et al.: Denosumab versus zoledronic acid in the prevention of skeletal-related events in vulnerable cancer patients: a meta-analysis of randomized, controlled trials. Clin Ther. 2020, 42:1494507.e1. 10.1016/j.clinthera.2020.05.019

74. Effing SM, Gyawali B: Assessing the risk-benefit profile of ramucirumab in patients with advanced solid tumors: a meta-analysis of randomized controlled trials. EClinicalMedicine. 2020, 25:100458. 10.1016/j.eclinm.2020.100458 